\title{
From offshore to onshore probabilistic tsunami hazard assessment via efficient Monte-Carlo sampling
}

\author{
Gareth Davies ${ }^{1}$, Rikki Weber ${ }^{1,2}$, Kaya Wilson $^{1}$, Phil Cummins ${ }^{1,2}$ \\ ${ }^{1}$ Place, Space and Community Division, Geoscience Australia, ACT, Australia \\ 2 Research School of Earth Sciences, Australian National University, ACT, Australia.
}

November 1, 2021

This manuscript is a non-peer-reviewed preprint submitted to EarthArXiv. At the time of writing it has been submitted to the journal Geophysical Journal International.

*gareth.davies@ga.gov.au 


\begin{abstract}
Offshore Probabilistic Tsunami Hazard Assessments (offshore PTHAs) provide large-scale analyses of earthquaketsunami frequencies and uncertainties in the deep ocean, but do not provide high-resolution onshore tsunami hazard information as required for many risk-management applications. To understand the implications of an offshore PTHA for the onshore hazard at any site, in principle the tsunami inundation should be simulated locally for every scenario in the offshore PTHA. In practice this is rarely feasible due to the computational expense of inundation models, and the large number of scenarios in offshore PTHAs. Monte-Carlo methods offer a practical and rigorous alternative for approximating the onshore hazard, using a random subset of scenarios. The resulting Monte-Carlo errors can be quantified and controlled, enabling high-resolution onshore PTHAs to be implemented at a fraction of the computational cost.

This study develops novel Monte-Carlo sampling approaches for offshore-to-onshore PTHA. Modelled offshore PTHA wave heights are used to preferentially sample scenarios that have large offshore waves near an onshore site of interest. By appropriately weighting the scenarios, the Monte-Carlo errors are reduced without introducing any bias. The techniques are applied to a high-resolution onshore PTHA for the island of Tongatapu in Tonga. In this region, the new approaches lead to efficiency improvements equivalent to using 4-18 times more random scenarios, as compared with stratified-sampling by magnitude, which is commonly used for onshore PTHA. The greatest efficiency improvements are for rare, large tsunamis, and for calculations that represent epistemic uncertainties in the tsunami hazard.

To facilitate the control of Monte-Carlo errors in practical applications, this study also provides analytical techniques for estimating the errors both before and after inundation simulations are conducted. Before inundation simulation, this enables a proposed Monte-Carlo sampling scheme to be checked, and potentially improved, at minimal computational cost. After inundation simulation, it enables the remaining Monte-Carlo errors to be quantified at onshore sites, without additional inundation simulations. In combination these techniques enable offshore PTHAs to be rigorously transformed into onshore PTHAs, with full characterisation of epistemic uncertainties, while controlling Monte-Carlo errors.
\end{abstract}

\title{
1 INTRODUCTION
}

Tsunami inundation is rare on most coastlines, but large events can have catastrophic impacts (Okal, 2011; Chock et al., 2013; Okal, 2015). Limited data is usually available to constrain the frequency of inundation, leading to uncertainties in the hazard that are consequential for risk analysis and management (Fukutani et al., 2018; Løvholt et al., 2019; Wood et al., 2020; Tonini et al., 2021). Probabilistic Tsunami Hazard Assessment (PTHA) offers a structured approach to quantifying the hazard and associated uncertainties, while integrating data, physical and statistical models, and expert judgements (Grezio et al., 2017; Behrens et al., 2021). Although PTHA is not yet standardised, several large-scale PTHAs provide analyses of offshore earthquake-tsunami frequencies and uncertainties at national-to-global scales (e.g. Burbidge et al., 2008, 2009; Chock, 2015; Power et al., 2017; Davies \& Griffin, 2018; Basili et al., 2021). Offshore PTHAs are well suited to representing diverse earthquake-tsunami sources at large spatial scales, but cannot accurately represent tsunami shoaling and inundation, so provide limited information on the onshore hazard. It is challenging to transform offshore PTHAs into high-resolution onshore PTHAs that give a similarly comprehensive representation of the hazard and uncertainty, while resolving spatial scales on the order of $10 \mathrm{~m}$ that are relevant to risk-management (Lynett et al., 2016). This is primarily because tsunami inundation modelling is computationally expensive, and a wide-range of scenarios are significant for characterising the hazard (Lorito et al., 2015; Sepúlveda et al., 2019; Gibbons et al., 2020; Tonini et al., 2021).

Recent offshore PTHAs represent hypothetical earthquake-tsunamis with a large set of scenarios $E$, containing on the order of $10^{5}-10^{7}$ individual scenarios $e \in E$ (Davies \& Griffin, 2020; Basili et al., 2021; Tonini et al., 2021). For each scenario the tsunami is simulated using computationally cheap models that are accurate in deep-water far from the coast, but inaccurate near the coast due to coarse resolution and neglect of nonlinearity (e.g. Burbidge et al., 2008; Miranda et al., 2014; Lynett et al., 2016; Molinari et al., 2016; Davies, 2019). Scenario-frequencies are represented using a family of models $I$ which collectively represent epistemic uncertainties in tsunami occurrence-rates, with each model $i \in I$ assigning a long-term average rate $r_{i}(e)$ (events/year) to every scenario (e.g. Power et al., 2012; Davies $\&$ Griffin, 2020; Basili et al., 2021). For any quantity of interest $Q$, such as the tsunami maxima at a particular site, this leads to a family of exceedance-rate curves describing the hazard and uncertainties. If scenarios do not overlap in time, the long-term average rate at which $Q$ exceeds a threshold $Q^{T}$ under scenario-frequency model $i$ is denoted $\lambda_{i}\left(Q>Q^{T}\right)$ (events/year) and:

$$
\lambda_{i}\left(Q>Q^{T}\right)=\sum_{e \in E} \mathbb{1}_{\left(Q(e)>Q^{T}\right)} r_{i}(e)
$$

Here $\mathbb{1}_{x}$ is the indicator function (=1 if $x$ is true and 0 otherwise). If the offshore PTHA describes an infinite set of scenarios then Equation 1 becomes an integral (Behrens et al., 2021). But herein we assume a large finite set of 
scenarios is used, and Equation 1 defines the exceedance-rate curves according to the offshore PTHA.

At coastal sites, the offshore PTHA is not designed to simulate the tsunami, but exceedance-rate curves can inprinciple be derived from Equation 1 by using a site-specific hydrodynamic model to compute $Q(e)$ for all scenarios. However for most onshore hazard assessments this is too computationally expensive, especially at large spatial scales. Recent studies using state-of-the-art high-performance-computing simulate tens of thousands of inundation scenarios over tens of kilometers of coastline at approximately $10 \mathrm{~m}$ resolution (Gibbons et al., 2020; Tonini et al., 2021), and most studies are conducted with far fewer computational resources. Furthermore a long-term goal of tsunami hazard research is the quantification of inundation hazards at much larger spatial scales (national or even global), for which the computational demands will be greater. Thus it is important to have good techniques to compute Equation 1, perhaps approximately, at lesser computational expense. This is the focus of the current study.

In hazard assessment practice, often a small number of scenarios are selected from the offshore PTHA, having tsunami maxima corresponding to a specified exceedance-rate (e.g. 1/500 or 1/2500) near the coastal site of interest (e.g. Boswood, 2013; Cardno, 2013; Chock, 2016). The exceedance-rates are often inferred from the mean scenariofrequency model or the $84^{\text {th }}$ percentile (Leonard et al., 2008; MCDEM, 2016; Tonini et al., 2021). This is a pragmatic approach when only a small number of inundation scenarios can be simulated. But there is no guarantee that the resulting inundation will closely approximate the 'ideal solution', derived from Equation 1 by brute-force computation (i.e. simulating inundation for every scenario). This is because modelled offshore wave heights are not monotonically related to modelled nearshore wave heights or inundation; relationships between these variables exhibit substantial scatter in practice (e.g. Tang et al., 2009; Mueller et al., 2015). For this reason the current study focusses on more rigorous approximations to Equation 1 at coastal sites, which enable the offshore PTHA to be translated onshore with controlled error, and at much lower cost than brute-force computation.

There are two complementary approaches to solving Equation 1 at coastal sites, and thus translating offshore PTHAs onshore:

1. Develop efficient methods to compute $Q(e)$ using high-performance-computing and/or approximation methods (e.g. de la Asunción et al., 2013; de Baar \& Roberts, 2017; Guillas et al., 2018; Arce Acuña \& Aoki, 2018; Williamson et al., 2020; Fukutani et al., 2021; Giles et al., 2021);

2. Develop techniques to approximate Equation 1 that require inundation simulations for only a small fraction of all scenarios (e.g. Lorito et al., 2015; Sepúlveda et al., 2017, 2019; Volpe et al., 2019).

While both are of fundamental importance to PTHA, this study focusses only on the second approach. We consider Monte-Carlo approximations of Equation 1 that only require simulating inundation for a (weighted) random subset of scenarios. Our approach builds upon stratified-sampling, in which scenarios are binned by magnitude and a fixed number are randomly sampled from each bin (e.g. De Risi \& Goda, 2017; Williamson et al., 2020; Basili et al., 2021; Zamora et al., 2021). This standard approach is improved by leveraging the offshore PTHA to automatically focus sampling on scenarios with larger waves near the site of interest, and on magnitude-bins that contribute most to Monte-Carlo errors.

Previously Lorito et al. (2015) and Volpe et al. (2019) developed techniques that leverage offshore PTHA waveheights to guide onshore PTHA scenario sampling. They firstly discarded scenarios that produced small waves offshore of the site of interest. The remaining scenarios were clustered by offshore wave-heights, with each cluster represented using one inundation simulation (Lorito et al., 2015). The clustering step was later refined to discard infrequent scenarios, and to reduce bias for near-field tsunamis (Volpe et al., 2019). Sepúlveda et al. (2017) also considered the problem of selecting a good subset of scenarios from among random earthquakes, although they did not assume the existence of offshore PTHA waveforms. Their Stochastic Reduced Order Method (SROM) enables selecting and weighting a subset of scenarios that collectively represents the variability of the co-seismic displacement. A case-study suggested this leads to a more efficient representation of the tsunami variability, compared with standard Monte-Carlo sampling (Sepúlveda et al., 2017).

Compared with previous works, our study places greater emphasis on simple analytical techniques to estimate errors caused by the limited sampling of scenarios, both before and after detailed inundation simulations are undertaken. This serves two purposes:

1. Before inundation simulation, it facilitates testing and refinement of Monte-Carlo sampling schemes. This enables poorly performing schemes to be identified and fixed, at minimal computational cost.

2. After inundation simulation, Monte-Carlo errors can be estimated at onshore sites, without additional simulations. Thus the numerical reliability of the onshore hazard can be easily quantified.

Used in combination, these techniques facilitate the control of Monte-Carlo errors in onshore hazard assessments. 
This paper is structured as follows. Section 2 introduces the site of interest and the offshore PTHA used to demonstrate our approach (Davies \& Griffin, 2018, 2020). Note our methodology is not specific to this offshore PTHA. Section 3 reviews common Monte-Carlo integration techniques for offshore-to-onshore PTHA: synthetic catalogues (Section 3.1) and stratified-sampling by magnitude (Section 3.2). Methods are presented to cheaply estimate the accuracy in coastal hazard applications, and we assess the computational cost of using many scenario-frequency models $i \in I$ to represent epistemic uncertainties. Section 3.3 extends the analysis to a novel Monte-Carlo integration technique, termed 'stratified/importance-sampling' herein, which leverages information from the offshore PTHA to better sample scenarios featuring larger waves near the site of interest. Section 3.4 develops techniques to guide non-uniform sampling of magnitude-bins, also informed by the offshore PTHA, which lead to additional efficiency improvements for both stratified and stratified/importance-sampling. Section 3.5 tests a particular combination of these approaches at offshore sites in the context of our inundation hazard application, which is presented in Section 4. Section 4.1 presents the high-resolution tsunami model and comparison with historic tsunamis at our site of interest, while Section 4.2 tests that Monte-Carlo results obtained from the high-resolution model are consistent with the offshore PTHA at a deep-ocean site. Section 4.3 examines onshore hazard results with a focus on the quantification of Monte-Carlo errors and epistemic uncertainties.

\section{STUDY SITE AND OFFSHORE PTHA}

To demonstrate Monte-Carlo techniques for offshore-to-onshore PTHA, we use offshore earthquake-tsunami scenarios and scenario-frequency models from the 2018 Australia Probabilistic Tsunami Hazard Assessment (henceforth PTHA18, Davies \& Griffin, 2018, 2020). Although focussed on Australia, PTHA18 includes results at globally distributed sites. Herein PTHA18 is used to model earthquake-tsunami inundation hazard in Tongatapu, the main island of Tonga (Figure 1A-B).

For simplicity our calculations are restricted to thrust earthquakes on the full Kermadec-Tonga source-zone (Figure 1A). This is the dominant source of large earthquake-generated tsunamis near Tongatapu according to the PTHA18 mean scenario-frequency model, producing $90 \%$ of tsunamis exceeding $2 \mathrm{~m}$ at Site P in Figure 1B. Instrumental and historical observations indicate that great $\left(M_{w}>8\right)$ tsunamigenic thrust earthquakes have occurred on the Kermadec-Tonga source-zone (e.g. Okal et al., 2004; Tang et al., 2008; Romano et al., 2021) and paleotsunami deposits are suggestive of older events (Goff et al., 2011; Power et al., 2012; Lamarche et al., 2015; Goff et al., 2020). However, Tongatapu is also exposed to other tsunami sources including outer-rise earthquakes (Lay et al., 2010), more complex local earthquakes (Okal et al., 2011), landslides and volcanic flank collapses (Frohlich et al., 2009; Goff, 2011), and far-field earthquakes. These are not treated herein and so our hazard results are incomplete, albeit sufficient for our primary purpose of illustrating Monte-Carlo techniques for offshore-to-onshore PTHA.

PTHA18 represents the Kermadec-Tonga thrust source-zone using a set of pure-thrust unit-sources (Figure 1A) with geometry based on SLAB2.0 (Hayes et al., 2018). Earthquake scenarios are represented as linear combinations of unit-sources, with magnitudes ranging from 7.2 to 9.6 in increments of 0.1. Although a range of uniform/heterogeneous slip earthquake models are included in PTHA18 and seem consistent with DART buoy observations (Davies, 2019; Davies \& Griffin, 2020), the hazard modelling herein exclusively uses heterogeneous-slip scenarios with constant rigidity. This is because those scenarios performed well in testing (Davies, 2019) and because many studies emphasise the importance of slip-heterogeneity in near-field settings (e.g. Geist, 2002; Mueller et al., 2015; Melgar et al., 2019), although we note that compact uniform-slip scenarios may also perform well (An et al., 2018; Davies, 2019).

On the Kermadec-Tonga trench, PTHA18 represents earthquake scenario-frequencies using an equally weighted combination of unsegmented and segmented representations. Three segments are distinguished for the latter; Tonga, Kermadec, and Hikurangi (Figure 1A), and their combination is termed the 'union-of-segments'. The segments do not represent hard-boundaries (i.e. earthquake scenarios can rupture on multiple segments), but represent the possibility that key source-zone parameters vary on each segment (e.g. maximum-magnitude, coupling, b-value) which the unsegmented model cannot. A family of 32,000 magnitude-frequency models are associated with each of the three segments and the unsegmented model (Figure 1C-F, details in Davies \& Griffin, 2020). The magnitudefrequency models are defined using a logic-tree and weighted using Bayesian techniques that leverage earthquake catalogue data, plate-convergence rates, and uncertain prior information on maximum magnitudes, coupling and the Gutenberg-Richter $b$ value (Davies \& Griffin, 2020). Each magnitude-frequency curve has an associated scenariofrequency model which partitions the seismicity among scenarios. This partition is non-uniform, even for scenarios with identical magnitudes, to obtain consistency with spatially variable plate convergence and adjust for model biases inferred by comparison with deep-ocean tsunamis (Davies \& Griffin, 2020).

The PTHA18 results imply large uncertainties in the frequency of high-magnitude earthquakes on the Kermadec- 

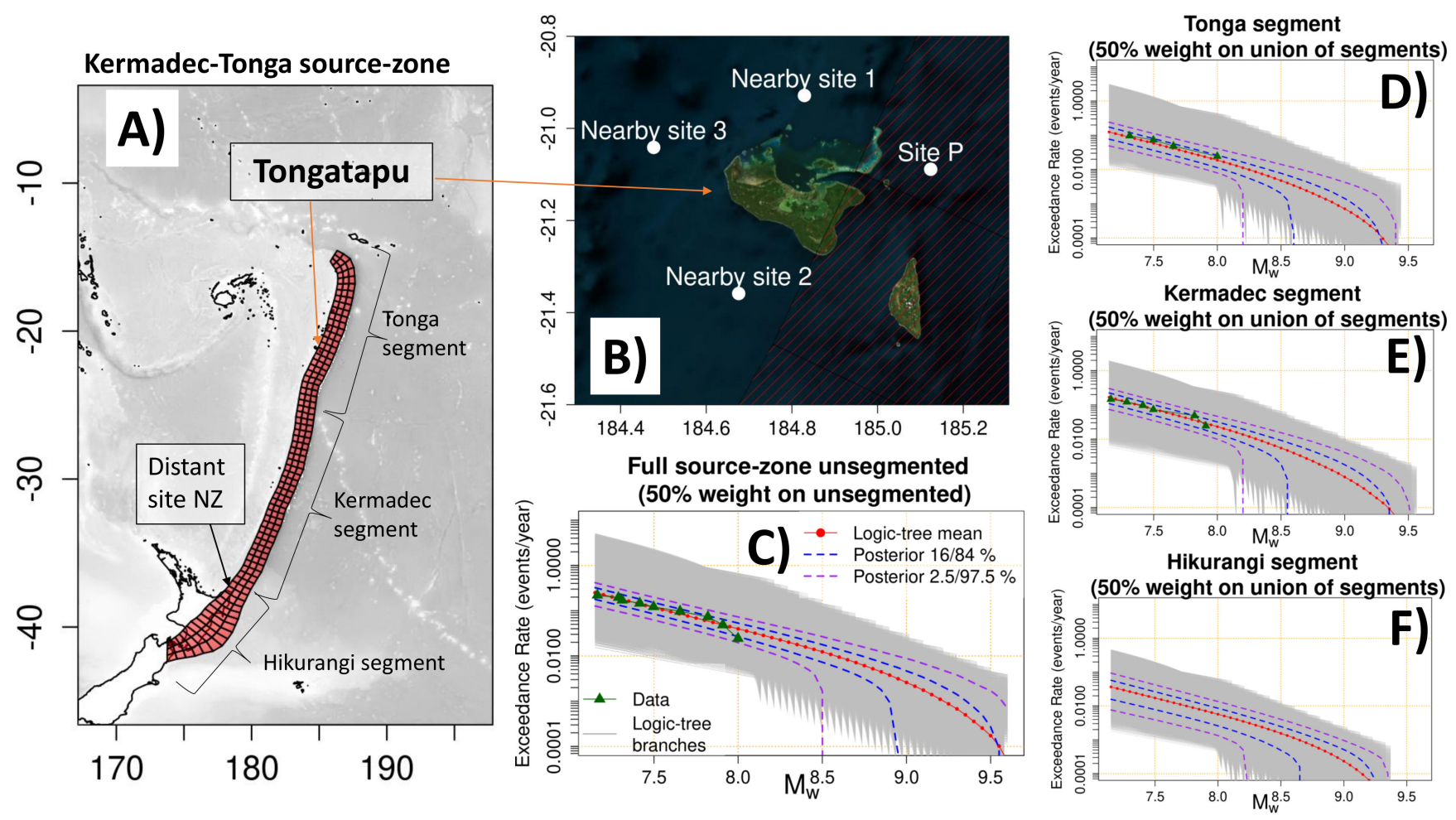

Figure 1: Tongatapu and PTHA18 magnitude-frequency models on the Kermadec-Tonga thrust source. A) The Kermadec-Tonga thrust source-zone as represented in PTHA18, with key sites featuring in this study. B) The island of Tongatapu, with four offshore sites from PTHA18 that are used in this study. C) The unsegmented magnitudefrequency model from PTHA18, which is given 50\% weight. Light-grey regions depict 32,000 alternative magnitudefrequency models that represent epistemic uncertainties in scenario-frequencies. Data show thrust-like earthquakes on the source-zone in 1976-2016, as used in PTHA18 (green triangles). The logic-tree mean and percentile curves summarise the alternative models and their weights. $D-F$ ) The segmented magnitude-frequency models used in PTHA18, which are similar to the unsegmented model but derived using the geometry and data for each segment. The union of these three segments is assigned $50 \%$ weight. 
Tonga trench (Figure 1C-F). This reflects the combination of a large area subduction interface (Hayes et al., 2018), high plate convergence rates (Bird, 2003), uncertain seismic coupling (Berryman et al., 2015), and a limited history of large subduction earthquakes (Okal et al., 2004, 2011). One interpretation is that there is relatively low coupling of the plate interface (Scholz \& Campos, 2012) and low maximum-magnitudes (Heuret et al., 2012; Bletery et al., 2016), in which case large tsunamigenic earthquakes may be relatively infrequent. However the earthquake history is far too short to preclude the possibility of earthquakes much larger than historically observed (McCaffrey, 2008; Zöller, 2013; Rong et al., 2014; Zöller, 2017). Such events have been suggested on the basis of paleotsunami evidence (Power et al., 2012; Goff et al., 2020) and seem plausible given the fault geometry (McCaffrey, 2008; Kalligeris et al., 2017; Davies et al., 2017; Power et al., 2017; Plescia \& Hayes, 2020). As a result there is much uncertainty as to the potential for large earthquakes on the Kermadec-Tonga trench (Berryman et al., 2015; UNESCO/IOC, 2020). This is reflected in the family of scenario-frequency models defined by PTHA18 and used herein.

\section{MONTE-CARLO INTEGRATION TECHNIQUES FOR OFFSHORE- TO-ONSHORE PTHA}

\subsection{Synthetic Catalogues}

In PTHA it is common for exceedance-rate curves (Equation 1) to be approximated at coastal sites by sampling a random synthetic catalogue with very long duration $D$ (years) from the offshore PTHA (e.g. Lane et al., 2012; Hoechner et al., 2016; Li et al., 2016b,a, 2018; Power et al., 2017). Below we analyse the Monte-Carlo accuracy of synthetic catalogues.

Denoting the set of scenarios in the synthetic catalogue as $E^{S C}$, exceedance-rates are estimated as:

$$
\widehat{\lambda_{i}^{S C}}\left(Q>Q^{T}\right)=\frac{1}{D}\left(\sum_{e \in E^{S C}} \mathbb{1}_{\left(Q(e)>Q^{T}\right)}\right)
$$

Here the hat notation $\widehat{\lambda_{i}^{S C}}\left(Q>Q^{T}\right)$ implies this is an estimate of the value of $\lambda_{i}\left(Q>Q^{T}\right)$ which is defined by Equation 1. The error in Equation 2 varies for each random synthetic catalogue; the term in large parenthesis has a mean of $\left(D \lambda_{i}\left(Q>Q^{T}\right)\right)$ and behaves like a Poisson random variable if earthquake occurrences are independent. Using standard results for the mean and variance of a Poisson random variable (e.g. Bolker, 2008) it follows that the average of $\widehat{\lambda_{i}^{S C}}\left(Q>Q^{T}\right)$ over all synthetic catalogues equals Equation 1, and their variance $\sigma^{2}$ is:

$$
\sigma^{2}\left(\widehat{\lambda_{i}^{S C}}\left(Q>Q^{T}\right)\right)=\lambda_{i}\left(Q>Q^{T}\right) / D
$$

which is the square of the standard deviation of the Monte-Carlo errors.

These results enable Monte-Carlo errors in Equation 2 to be constrained prior to sampling, which can help modellers determine a suitable catalogue duration. Suppose a synthetic catalogue with duration $D=100,000$ years is used (e.g. Lane et al., 2012; Li et al., 2016b; Power et al., 2017; Li et al., 2018). If Equation 1 gives an exceedance-rate of 1/1000, then $95 \%$ of Monte-Carlo estimates will be within the interval $(1 / 1235,1 / 833)$ derived using a Poisson distribution, or approximately $\pm 20 \%$ (using Equations 2-3 and assuming a 2-standard-deviation interval). The typical error increases for rare events; if Equation 1 gives an exceedance-rate of 1/10000, then $95 \%$ of Monte-Carlo estimates will be in $(1 / 25000,1 / 5882)$, or approximately $\pm 63 \%$. The acceptability of these errors will vary from case-to-case; while they should be minimised for numerical accuracy, it may be practically acceptable if they are small relative to epistemic uncertainties in the exceedance-rates (Li et al., 2016b; Power et al., 2017).

Synthetic catalogues are often inefficient for tsunami inundation hazard calculations (Sepúlveda et al., 2019; Williamson et al., 2020). If seismicity follows a Gutenberg-Richter like distribution then they tend to be dominated by smaller magnitudes, while larger magnitude scenarios are resolved less well despite often being more relevant in tsunami hazard applications. However if only large scenarios are of interest, this can be partly mitigated by ignoring smaller scenarios (Lane et al., 2012).

Another inefficiency arises if multiple scenario-frequency models $i \in I$ are used to represent epistemic uncertainties. Because the scenario-frequency is represented by the catalogue itself, in general different synthetic catalogues are required to represent each model $i \in I$ (or at least a sufficiently large random sample of scenario-frequency models should be used, e.g. Power et al. (2017)). This leads to multiplicative growth in the number of scenarios, and simulating these through to inundation may be prohibitively expensive. 


\subsection{Stratified-Sampling by Magnitude}

\subsubsection{Sampling approach and statistical properties of the Monte-Carlo error}

To improve on the efficiency of synthetic catalogues, PTHAs often employ stratified-sampling by earthquake magnitude (e.g. De Risi \& Goda, 2017; Williamson et al., 2020; Basili et al., 2021; Zamora et al., 2021). The Monte-Carlo accuracy of this approach is analysed below, and techniques are presented to estimate the errors both before and after high-resolution tsunami simulation.

For stratified-sampling the PTHA scenarios are grouped into discrete magnitude-bins $M_{w, b}$. For example the current study uses uniformly-spaced bins inherited from PTHA18:

$$
M_{w, b} \in\{(7.15,7.25],(7.25,7.35], \ldots,(9.55,9.65]\}
$$

On some scenario-frequency models $i \in I$ the higher magnitude bins will be impossible (zero occurrence-rate) depending on the maximum-magnitude for model $i$ (details in Davies \& Griffin, 2020).

From the offshore PTHA, the occurrence-rate of scenarios in magnitude-bin $M_{w, b}$ is known:

$$
\lambda_{i}\left(M_{w, b}\right)=\sum_{e \in E_{b}} r_{i}(e)
$$

Here $E_{b}$ is the set of all PTHA scenarios in magnitude-bin $M_{w, b}$. To approximate the exceedance-rate (Equation 1) a number $N\left(M_{w, b}\right)$ of scenarios are sampled from $E_{b}$ using weighted random sampling with replacement. For stratifiedsampling, if $M_{w, b}$ has non-zero occurrence-rate then the chance of sampling each scenario $w_{b, i}^{S S}(e)$ must equal the within-magnitude-bin scenario conditional probability:

$$
w_{b, i}^{S S}(e)=\frac{r_{i}(e)}{\sum_{e \in E_{b}} r_{i}(e)}
$$

Note the novel sampling scheme proposed in Section 3.3 provides a more flexible analogue of Equation 6 .

Denote the set of randomly sampled scenarios in magnitude-bin $M_{w, b}$ as $E_{b, i}^{S S}$. Suppose the tsunami has been simulated for each. The within-magnitude-bin exceedance-rate may be estimated as:

$$
\widehat{\lambda_{i}^{S S}}\left(Q>Q^{T} \mid M_{w, b}\right)=\lambda_{i}\left(M_{w, b}\right)\left(\sum_{e \in E_{b, i}^{S S}} \mathbb{1}_{\left(Q(e)>Q^{T}\right)}\right) / N\left(M_{w, b}\right)
$$

This varies with the random sample $E_{b, i}^{S S}$. The term in large parenthesis in Equation 7 behaves like a binomial random variable with size $N\left(M_{w, b}\right)$ and probability $p_{b, i, T}$, where:

$$
p_{b, i, T}=\frac{\sum_{e \in E_{b}} r_{i}(e) \mathbb{1}_{\left(Q(e)>Q^{T}\right)}}{\sum_{e \in E_{b}} r_{i}(e)}
$$

is the probability that a random PTHA scenario in $E_{b}$ exceeds the threshold $Q^{T}$. Using standard formulae for the mean and variance of a binomial random variable (e.g. Bolker, 2008) it follows that Equation 7 gives an unbiased estimate of the within-magnitude-bin exceedance-rate and has variance:

$$
\sigma^{2}\left(\widehat{\lambda_{i}^{S S}}\left(Q>Q^{T} \mid M_{w, b}\right)\right)=\frac{1}{N\left(M_{w, b}\right)}\left(\lambda_{i}\left(M_{w, b}\right)\right)^{2} p_{b, i, T}\left(1-p_{b, i, T}\right)
$$

Calculation of the Monte-Carlo variance (Equation 9) requires that $Q(e)$ is known for all $e \in E_{b}$, to evaluate $p_{b, i, T}$ in Equation 8. This is possible at offshore sites (using the offshore PTHA) but generally impossible at coastal sites. However an estimate of the variance at coastal sites can be derived after the tsunami has been simulated for all random scenarios in $E_{b, i}^{S S}$, via an estimate of $p_{b, i, T}\left(\right.$ denoted $\left.\widehat{p}_{b, i, T}\right)$.

$$
\begin{aligned}
\widehat{p}_{b, i, T} & =\left(\sum_{e \in E_{b, i}^{S S}} \mathbb{1}_{\left(Q(e)>Q^{T}\right)}\right) / N\left(M_{w, b}\right) \\
\widehat{\sigma^{2}}\left(\widehat{\lambda_{i}^{S S}}\left(Q>Q^{T} \mid M_{w, b}\right)\right) & =\frac{1}{N\left(M_{w, b}\right)}\left(\lambda_{i}\left(M_{w, b}\right)\right)^{2} \widehat{p}_{b, i, T}\left(1-\widehat{p}_{b, i, T}\right)
\end{aligned}
$$


Summation of Equation 7 over magnitude-bins leads to an unbiased estimate of the exceedance-rate (Equation 1) which is of primary interest for PTHA:

$$
\widehat{\lambda_{i}^{S S}}\left(Q>Q^{T}\right)=\sum_{M_{w, b} \in \text { magnitude-bins }} \widehat{\lambda_{i}^{S S}}\left(Q>Q^{T} \mid M_{w, b}\right)
$$

This will vary for different Monte-Carlo samples. Because random samples in different magnitude-bins are independent, the variance of Equation 11 is the sum of the within-magnitude-bin variances (Equation 9):

$$
\sigma^{2}\left(\widehat{\lambda_{i}^{S S}}\left(Q>Q^{T}\right)\right)=\sum_{M_{w, b} \in \text { magnitude-bins }} \sigma^{2}\left(\widehat{\lambda_{i}^{S S}}\left(Q>Q^{T} \mid M_{w, b}\right)\right)
$$

Equation 12 is termed the analytical Monte-Carlo variance herein because it is computed without Monte-Carlo sampling (at sites where the offshore PTHA is valid). This offers a useful check on stratified-sampling schemes in coastal hazard applications, prior to sampling and running expensive tsunami models. For example if the analytical standard-deviation (i.e. square-root of Equation 12) is a high-fraction of the exceedance-rate (Equation 1) at an offshore site, then we expect nearby coastal-hazard estimates will also be inaccurate and may decide to revise the sampling scheme. Although the Monte-Carlo variance can also be computed empirically (from the variance of repeated Monte-Carlo samples), Equation 12 is faster and simpler. It also plays a core role in the design of non-uniform sampling strategies (Section 3.4).

The analytical Monte-Carlo variance (Equation 12) cannot be computed at coastal sites because $Q(e)$ is not known for all $e \in E$. However it can be estimated after scenarios are sampled and inundation simulated, by summation over Equation 10:

$$
\widehat{\sigma^{2}}\left(\widehat{\lambda_{i}^{S S}}\left(Q>Q^{T}\right)\right)=\sum_{M_{w, b} \in \text { magnitude-bins }} \widehat{\sigma^{2}}\left(\widehat{\lambda_{i}^{S S}}\left(Q>Q^{T} \mid M_{w, b}\right)\right)
$$

This single-Monte-Carlo-sample variance estimate is useful at coastal sites, where in practice $Q(e)$ is only known for the random scenarios. Equation 13 enables the numerical reliability of the Monte-Carlo exceedance-rate to be estimated, without additional simulations.

To test this Monte-Carlo approach we consider the tsunami maximum-stage exceedance-rates at Site P, located in deep-water east of Tongatapu (Figure 1B). Only thrust earthquakes on the Kermadec-Tonga source-zone are considered. At Site P the exceedance-rates are defined by the offshore PTHA (Equation 1) and can be evaluated without Monte-Carlo sampling; this enables the Monte-Carlo estimates to be tested against known values. Scenario rates $r_{i}(e)$ were determined from the logic-tree mean scenario-frequency model $\bar{r}(e)$ in PTHA18, which has 50:50 weight on the unsegmented and union-of-segments models (Figure 1). Epistemic uncertainties in scenario-frequencies are ignored for simplicity (but considered later).

Ten-thousand Monte-Carlo exceedance-rate curves were computed at Site P (Figure 2). Each Monte-Carlo sample uses 1200 random scenarios, with $N\left(M_{w}\right)=48$ in each magnitude-bin (Equation 4). This corresponds to a computationally feasible number of inundation simulations for our case-study (Section 4) and is constrained by model run-times and computational resources.

The variability of the Monte-Carlo exceedance-rates is visually clear (Figure 2). To compare this stratifiedsampling technique with synthetic catalogues, the equivalent synthetic catalogue duration $D$ was defined such that 1200 random scenarios are included on average (implying an equivalent computational effort for inundation calculations). Comparison of $95 \%$ intervals for the synthetic catalogue and stratified-sampling confirms that errors in the latter grow more slowly at rare return periods, which shows the benefit of magnitude stratification (Figure 2).

To highlight the validity of the Monte-Carlo technique and the analytical and single-Monte-Carlo-sample variance calculations, consider the stage-threshold $Q^{T}=2 \mathrm{~m}$ (Figure 2). At this threshold the exceedance-rate in Equation 1 differs by only $0.2 \%$ from the average of the Monte-Carlo estimates computed with Equation 11 . Furthermore the empirical standard-deviation of the 10,000 Monte-Carlo estimates differs by only $0.3 \%$ from the analytical standarddeviation (square root of Equation 12). The distribution of the Monte-Carlo results can be approximated with a normal distribution having mean and variance equal to Equations 1 and 12, both of which are computed without Monte-Carlo sampling (Figure 2). This was used to compute the $95 \%$ confidence-intervals in Figure 2 . The key point is that, at offshore sites, we can quantify the performance of stratified-sampling before conducting Monte-Carlo simulation. This enables the sampling scheme performance to be checked and perhaps further optimised, prior to expensive tsunami simulation.

At coastal sites the offshore PTHA cannot accurately represent the tsunami, so $Q(e)$ is only known for a single Monte-Carlo sample (1200 scenarios) after tsunami simulations are completed. In that case Equations 11 and 13 

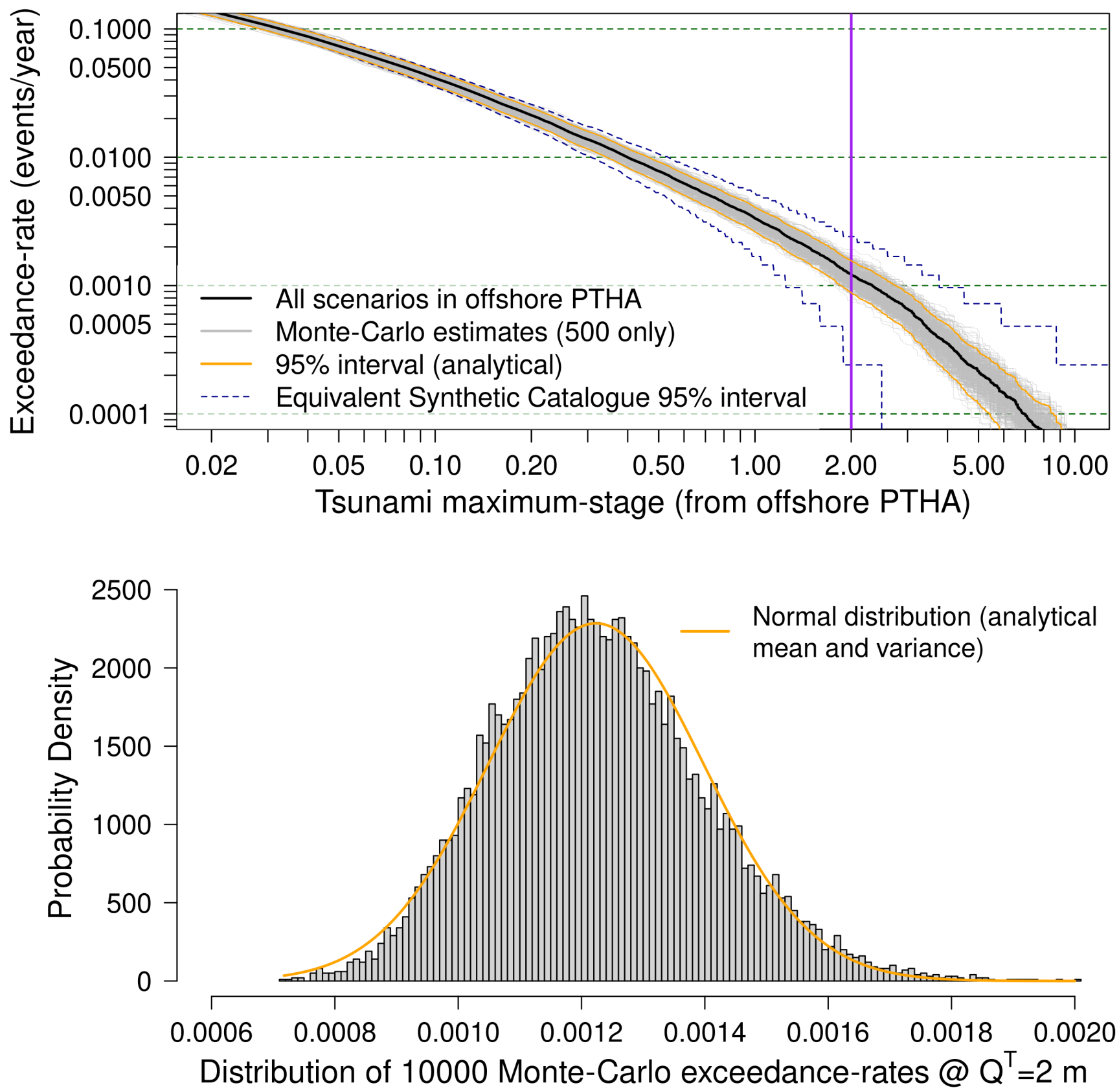

Figure 2: Performance of stratified-sampling at offshore Site P (location in Figure 1) for PTHA18 thrust scenarios on the Kermadec-Tonga trench. Top: Logic-tree mean exceedance-rate curve and Monte-Carlo estimates. Grey curves depict 500 of the 10,000 Monte-Carlo estimates. Bottom: Histogram of the 10,000 Monte-Carlo exceedance-rates for $Q^{T}=2 \mathrm{~m}$, compared with a normal distribution having mean and variance derived from Equations 1 and 12 respectively. 
provide a single-Monte-Carlo-sample estimate of the exceedance-rate and its Monte-Carlo variance, and an approximate $95 \%$ confidence-interval for the exceedance-rate in Equation 1 can be constructed by substitution into a normal distribution:

$$
\widehat{\lambda_{i}^{S S}}\left(Q>Q^{T}\right) \pm 1.96 \sqrt{\widehat{\sigma^{2}}\left(\widehat{\lambda_{i}^{S S}}\left(Q>Q^{T}\right)\right)}
$$

If Monte-Carlo sampling is repeated then this interval will cover the exceedance-rate in Equation 1 approximately, but not exactly, $95 \%$ of the time. For the 10,000 Monte-Carlo samples in Figure 2, the empirical coverage was $92.65 \%$ at $Q^{T}=2 \mathrm{~m}$. While approximate, this gives a reasonable indication of the Monte-Carlo error at coastal sites when only a single Monte-Carlo sample is available.

\subsubsection{Stratified-sampling with epistemic uncertainties}

How efficient is stratified-sampling when epistemic uncertainties are represented with a family of scenario-frequency models $i \in I$ ? Recall synthetic catalogues are inefficient in this context because the scenario-frequencies are represented by the catalogue itself, so a new catalogue is required for each $i \in I$ (Section 3.1). In contrast, for stratified-sampling it is the within-magnitude-bin scenario conditional probabilities that are represented by the catalogue itself (via Equation 6). Thus a new stratified-sample should be constructed for each variation of Equation 6 among the scenario-frequency models $i \in I$.

Fortunately, in practice PTHA methodologies often assume the within-magnitude-bin scenario conditional probabilities are independent of the particular scenario-frequency model, which facilitates the re-use of stratified-samples. This holds for PTHAs that assume all scenarios within a magnitude-bin are equally likely (e.g. Lorito et al., 2015; Williamson et al., 2020). It also holds for the approach of Davies \& Griffin (2020) which uses non-uniform conditional probabilities to represent spatial variations in tectonics and correct for earthquake model biases. For PTHA18, the within-magnitude-bin scenario conditional probabilities are identical for all 32,000 scenario-frequency models within the unsegmented model (Figure 1C), and separately, within each of the three segments (Figures 1D-F). These four source models share the same scenarios but with different within-magnitude-bin scenario conditional probabilities (full details in Davies \& Griffin, 2018, 2020). Scenario sharing is possible because, for example, a scenario on the Tonga segment can also occur on the unsegmented model, and a rupture crossing segment boundaries receives a partial occurrence-rate from each included segment. However a scenario that is fully contained in one segment will have zero occurrence-rate on the other segments. Thus the within-magnitude-bin scenario conditional probabilities must vary between segments. To represent this with stratified-sampling, four different versions of Equation 6 must be considered: Unsegmented, Tonga segment, Kermadec segment, and Hikurangi segment.

Thus for this study, epistemic uncertainty calculations via stratified-sampling require four sets of random scenarios. Each corresponds to a different panel in Figure 1C-F, and the 1200 scenarios must be partitioned among these; within each the Monte-Carlo sample can be re-used for every scenario-frequency model. The use of four sets of scenarios represents a big improvement over synthetic catalogues, which would require a separate catalogue for every $i \in I$, or at least a large random sample of those models (Power et al., 2017).

\subsection{Improving the efficiency near a site of interest: stratified/importance-sampling informed by offshore wave heights}

\subsubsection{Sampling approach and statistical properties of the Monte-Carlo error}

This section develops a novel 'stratified/importance-sampling' scheme for offshore-to-onshore PTHA which is more efficient than stratified-sampling near a specified site of interest, but potentially less efficient far from that site. As with stratified-sampling, techniques are presented to estimate the Monte-Carlo errors both before and after tsunami inundation simulation.

Our site of interest is exposed to a large earthquake source-zone (Figure 1A,B) and for scenarios within a magnitude-bin, the tsunami impacts will vary greatly with the earthquake location and slip-distribution. Suppose a rough estimate of the 'scenario importance' for our site can be derived from the offshore PTHA, without inundation computation. Improved Monte-Carlo exceedance-rates might be obtained by preferentially sampling more important scenarios, with appropriate weighting to prevent bias. The theory of importance-sampling (Tokdar \& Kass, 2009) provides a framework for achieving this. The approach herein is termed 'stratified/importance-sampling' because scenarios are also stratified by magnitude.

The method requires a user-defined notion of the 'scenario importance'. This is a positive number $\mathcal{I}(e)$ associated with every scenario $e \in E$; in each magnitude-bin the chance of randomly sampling a scenario will be rescaled in 
proportion to $\mathcal{I}(e)$. This study uses the PTHA18 maximum-stage at Site P (Figure 1B):

$$
\mathcal{I}(e)=\text { PTHA18 maximum-stage at point } P \text { for scenario } e
$$

Alternative definitions of $\mathcal{I}(e)$ are not explored here; good choices should be indicative of inundation potential at the site of interest, easy to compute, and avoid reference to a specific scenario-frequency model. It is possible to make a poor choice of $\mathcal{I}(e)$ which reduces the accuracy compared with stratified-sampling. However that risk can be mitigated by testing the approach at nearby offshore sites, before inundation computation (discussed below).

Suppose that scenarios are grouped into discrete magnitude-bins $M_{w, b}$, and a user-specified number $N\left(M_{w, b}\right)$ are sampled per-bin using weighted random sampling with replacement. In contrast to stratified-sampling, the chance $w_{b, i}^{S I S}(e)$ of sampling each scenario is:

$$
w_{b, i}^{S I S}(e)=\frac{\mathcal{I}(e) r_{*}(e)}{\sum_{e \in E_{b}} \mathcal{I}(e) r_{*}(e)}
$$

This is identical to standard stratified-sampling if $\mathcal{I}(e)$ is constant and $r_{*}(e)=r_{i}(e)$ (Equation 6). If only one model $i \in I$ is considered then the function $r_{*}(e)$ should equal $r_{i}(e)$, but other choices are useful when treating epistemic uncertainties in scenario-frequencies (Section 3.3.2). Any choice of $r_{*}(e)$ should satisfy $r_{*}(e)>0$ whenever $r_{i}(e)>0$, to retain a non-zero chance of sampling scenario $e$.

Using the random scenarios in magnitude-bin $M_{w, b}$ (denoted $\left.E_{b, i}^{S I S}\right)$ the within-magnitude-bin exceedance-rate is estimated as:

$$
\widehat{\lambda_{i}^{S I S}}\left(Q>Q^{T} \mid M_{w, b}\right)=\lambda_{i}\left(M_{w, b}\right)\left(\sum_{e \in E_{b, i}^{S I S}} \phi_{b, i}^{S I S}(e) \mathbb{1}_{\left(Q(e)>Q^{T}\right)}\right) / N\left(M_{w, b}\right)
$$

This is similar to stratified-sampling (Equation 7) except importance-sampling weights $\phi_{b, i}^{S I S}(e)$ appear, to correct for the biased sampling. Using the approach of 'basic importance-sampling' (e.g. Owen \& Zhou, 2000; Tokdar \& Kass, 2009) these weights are:

$$
\phi_{b, i}^{S I S}(e)=\left(\frac{r_{i}(e)}{\sum_{e \in E_{b}} r_{i}(e)}\right) / w_{b, i}^{S I S}(e)
$$

For impossible scenarios $\left(r_{*}(e)=0\right)$ Equation 18 involves zero division so is undefined; these cases are set zero.

Basic importance-sampling is unbiased (e.g. Owen \& Zhou, 2000; Tokdar \& Kass, 2009) thus Equation 17 is on average equal to the within-magnitude-bin exceedance-rate. It varies with the random sample $E_{b, i}^{S I S}$ and is asymptotically normal as $N\left(M_{w, b}\right) \rightarrow \infty$, with variance (Geweke, 1989; Tokdar \& Kass, 2009):

$$
\sigma^{2}\left(\widehat{\lambda_{i}^{S I S}}\left(Q>Q^{T} \mid M_{w, b}\right)\right)=\frac{\left(\lambda_{i}\left(M_{w, b}\right)\right)^{2}}{N\left(M_{w, b}\right)} \sum_{e \in E_{b}}\left(\left[\mathbb{1}_{\left(Q(e)>Q^{T}\right)} \phi_{b, i}^{S I S}(e)-p_{b, i, T}\right]^{2} w_{b, i}^{S I S}(e)\right)
$$

Equation 19 is analogous to Equation 9 for stratified-sampling, and is identical if $w_{b, i}^{S S}(e)=w_{b, i}^{S I S}(e)$. In both cases the analytical variance computation does not require any Monte-Carlo sampling, but does require that $Q(e)$ is known for all scenarios in $E_{b}$ which prevents its use at coastal sites (where the offshore PTHA does not accurately model the tsunami). But as with stratified-sampling, an estimate of the variance can be derived using a single Monte-Carlo sample (Tokdar \& Kass, 2009):

$$
\widehat{\sigma^{2}}\left(\widehat{\lambda_{i}^{S I S}}\left(Q>Q^{T} \mid M_{w, b}\right)\right)=\frac{\left(\lambda_{i}\left(M_{w, b}\right)\right)^{2}}{N\left(M_{w, b}\right)} \sum_{e \in E_{b, i}^{S I S}}\left(\left[\mathbb{1}_{\left(Q(e)>Q^{T}\right)} \phi_{b, i}^{S I S}(e)-\widehat{q}_{b, i, T}\right]^{2} / N\left(M_{w, b}\right)\right)
$$

where:

$$
\widehat{q}_{b, i, T}=\left(\sum_{e \in E_{b, i}^{S I S}} \phi_{b, i}^{S I S}(e) \mathbb{1}_{\left(Q(e)>Q^{T}\right)}\right) / N\left(M_{w, b}\right)
$$

is an estimate of $p_{b, i, T}$ (Equation 8).

Analogues of key equations for stratified-sampling (Equations 11-13) follow. Summation of Equation 17 over magnitude-bins gives an unbiased estimate of the exceedance-rate in Equation 1:

$$
\widehat{\lambda_{i}^{S I S}}\left(Q>Q^{T}\right)=\sum_{M_{w, b} \in \text { Magnitude bins }} \widehat{\lambda_{i}^{S I S}}\left(Q>Q^{T} \mid M_{w, b}\right)
$$


The analytical variance of this estimate is the sum of the within magnitude-bin variances (Equation 19):

$$
\sigma^{2}\left(\widehat{\lambda_{i}^{S I S}}\left(Q>Q^{T}\right)\right)=\sum_{M_{w, b} \in \text { magnitude-bins }} \sigma^{2}\left(\widehat{\lambda_{i}^{S I S}}\left(Q>Q^{T} \mid M_{w, b}\right)\right)
$$

Equation 23 enables the Monte-Carlo exceedance-rate variance to be calculated prior to sampling (at offshore sites), facilitating checks of the sampling scheme prior to inundation simulation.

Although Equation 23 cannot generally be computed at coastal sites, the variance can be estimated when tsunami simulations are completed for a single-Monte-Carlo-sample by summation over Equation 20:

$$
\widehat{\sigma^{2}}\left(\widehat{\lambda_{i}^{S I S}}\left(Q>Q^{T}\right)\right)=\sum_{M_{w, b} \in \text { magnitude-bins }} \widehat{\sigma^{2}}\left(\widehat{\lambda_{i}^{S I S}}\left(Q>Q^{T} \mid M_{w, b}\right)\right)
$$

To demonstrate the improved accuracy, Figure 3 repeats the Monte-Carlo test at Site P using stratified/importancesampling. Because the previous stratified-sampling results apply to the logic-tree mean scenario frequency model $\bar{r}(e)$, for stratified/importance-sampling we set $r_{*}(e)=\bar{r}(e)$ in Equation 16. Compared with stratified-sampling (Figure 2) the reduction in the Monte-Carlo error is visually obvious; for example at $Q^{T}=2 \mathrm{~m}$ the empirical standard deviation of the Monte-Carlo exceedance-rates is 2.45 times smaller. To achieve similar accuracy with stratifiedsampling would require using $2.45^{2}=6$ times as many scenarios (7200 vs 1200) and thus a factor of 6 increase in the computational effort.

In this example the average of the 10,000 Monte-Carlo exceedance-rates at $Q^{T}=2 \mathrm{~m}$ differs from Equation 1 by only $0.05 \%$, reflecting the lack of bias. The analytical standard-deviation (square root of Equation 23) agrees with the Monte-Carlo standard-deviation within $0.6 \%$, confirming the accuracy of the analytical variance (Figure 3 ). Furthermore, a single-Monte-Carlo-sample approximate confidence-interval for the exceedance-rate (Equation 1) can be derived as for Equation 14, which is particularly useful at coastal sites:

$$
\widehat{\lambda_{i}^{S I S}}\left(Q>Q^{T}\right) \pm 1.96 \sqrt{\widehat{\sigma^{2}}\left(\widehat{\lambda_{i}^{S I S}}\left(Q>Q^{T}\right)\right)}
$$

For the 10,000 Monte-Carlo samples in Figure 3 this approximate confidence-interval included the Equation 1 exceedance-rate $94.55 \%$ of the time, very close to the ideal value of $95 \%$. These results confirm the utility of the analytical and single-Monte-Carlo-sample estimates of the Monte-Carlo exceedance-rate variance.

Crucially, the improved Monte-Carlo efficiency is not restricted to Site P that was used to define the scenario importance. If the tsunami is large at Site $\mathrm{P}$ it also tends to be large at nearby sites, which also show reductions in the Monte-Carlo variance (see Nearby sites 1, 2 and 3 in Figure 4, with locations in Figure 1B). The ratio of the Monte-Carlo variances associated with each sampling method, denoted VR and shown in Figure 4, is equivalent to the increase in computational effort required to make stratified-sampling as accurate as stratified/importancesampling. The VR values in Figure 4 were computed analytically (Equation 12/Equation 23), but very similar values were obtained using empirical variances of the Monte-Carlo samples (within 3.1\%). At sites near to Site P, VR is comparable to that which would be obtained by using each site's tsunami maxima to define its own $\mathcal{I}(e)$ (shown in parenthesis in panels of Figure 4). Efficiency improvements are also expected at the onshore site of interest (Tongatapu) because it is near Site P; this is the core idea behind stratified/importance-sampling.

While performing well near to Site $\mathrm{P}$, the definition of $\mathcal{I}(e)$ will not give a good proxy of the tsunami size far from Site P. While the method remains valid, the Monte-Carlo error can increase relative to stratified-sampling. For example this occurs at 'Distant site NZ' about $2000 \mathrm{~km}$ south of Site P (Figure 4, see site location in Figure 1A). If the goal is to conduct a hazard assessment near Site $\mathrm{P}$ then the reduced accuracy at distant sites is of little concern. If the goal were to conduct a hazard assessment near 'Distant site NZ', then Site P is not a good choice for defining the scenario importance $\mathcal{I}(e)$. For example at 'Distant site NZ', a factor 8.98 efficiency improvement could be obtained with a local definition of $\mathcal{I}(e)$ (Figure 4).

These results suggest stratified/importance-sampling based on Site P will be useful throughout the Tongatapu region. We recommend conducting similar checks in coastal hazard applications, prior to running expensive tsunami simulations. With a poor choice of $\mathcal{I}(e)$ importance-sampling might perform badly (like at Distant Site NZ, Figure 4)

or even fail (e.g. if the tails of the distribution are poorly represented, Owen \& Zhou, 2000). In the context of PTHA this would result in poor performance at nearby offshore sites, and can thus be detected and fixed at little computational cost.

\subsubsection{Stratified/importance sampling with epistemic uncertainties}

Can a single Monte-Carlo sample obtained with stratified/importance-sampling be re-used for epistemic uncertainty calculations? The key issue is whether the chance of sampling any scenario (Equation 16) is unchanged for multiple 

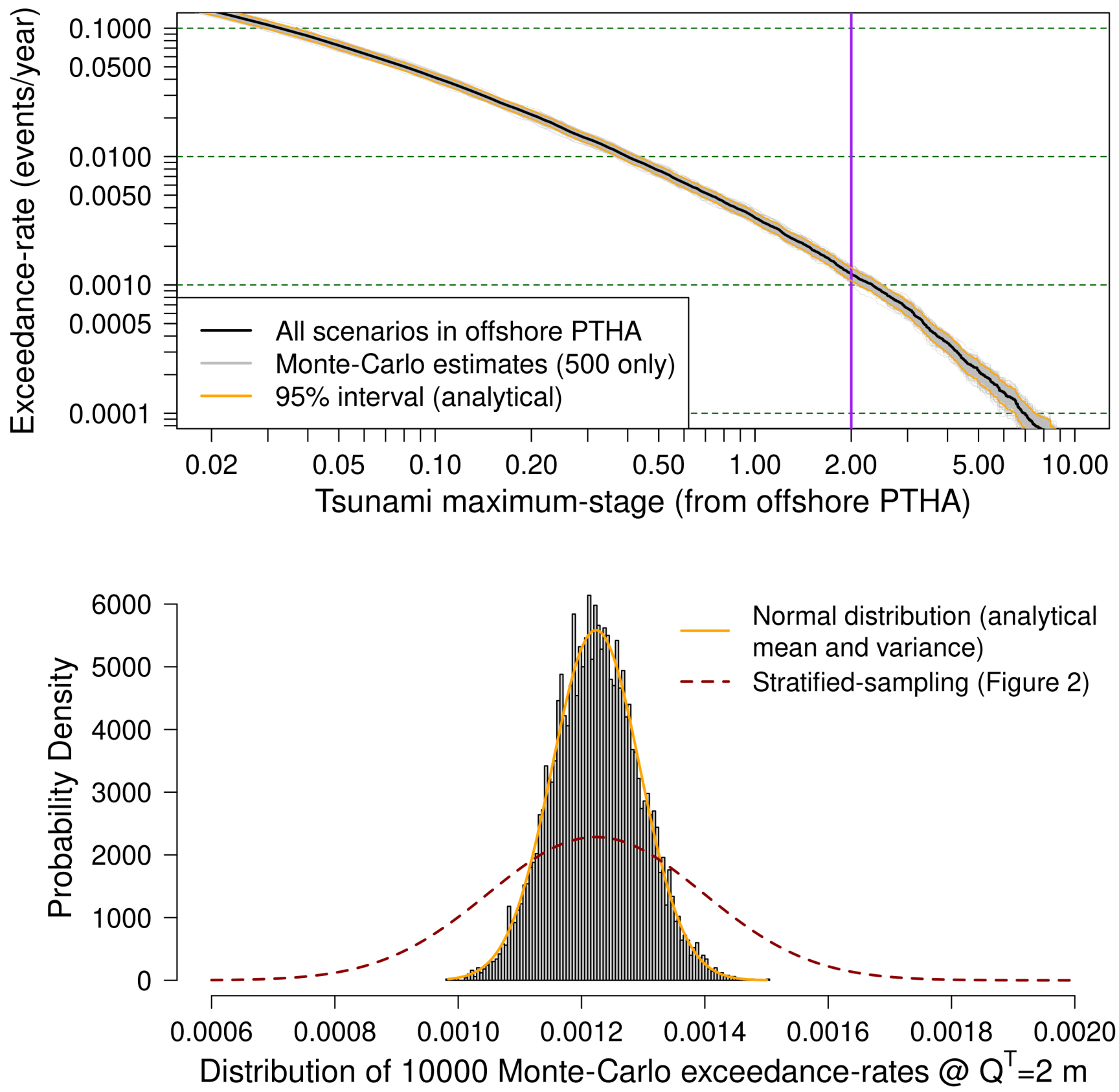

Figure 3: Performance of stratified/importance-sampling at an offshore Site P (location in Figure 1) for PTHA18 thrust scenarios on the Kermadec-Tonga trench. Top: Logic-tree mean exceedance-rate curve and Monte-Carlo estimates. Grey curves depict 500 of the 10,000 Monte-Carlo. Bottom: Histogram of the 10,000 Monte-Carlo exceedance-rates for $Q^{T}=2 \mathrm{~m}$, compared with a normal distribution having mean and variance derived from Equations 1 and Equation 23 respectively. The dashed-line gives the normal distribution derived via stratifiedsampling (see bottom-panel of Figure 2) 

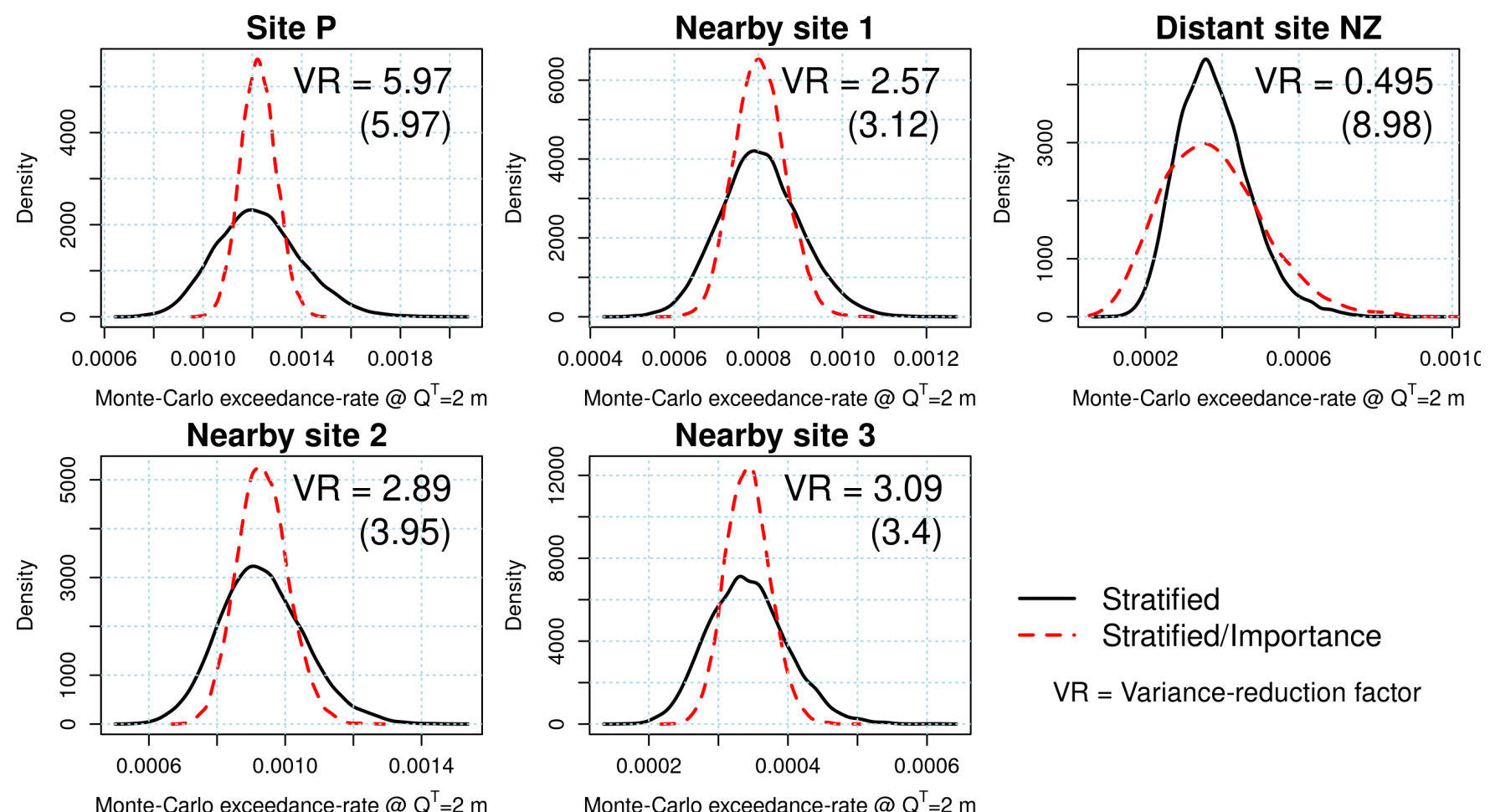

$\mathrm{VR}=$ Variance-reduction factor

Figure 4: Variability of Monte-Carlo exceedance-rate estimates for $Q^{T}=2 \mathrm{~m}$ using stratified and stratified/importance-sampling (10,000 Monte-Carlo samples, each with 1200 scenarios). Site locations shown in Figure 1A-B. VR gives the ratio of the Monte-Carlo variances with stratified and stratified/importance-sampling. Site $\mathrm{P}$ was used to define the scenario importance in all cases; but numbers in parenthesis show the VR value that would be obtained if the site's own tsunami-maxima were used instead. All calculations use the logic-tree mean scenario-frequency model. 
scenario-frequency models $i \in I$. Section 3.2.1 showed that for stratified-sampling, the chance of sampling each scenario (Equation 6) is equal to the within-magnitude-bin scenario conditional probability, necessitating four sets of stratified-samples in the context of this study (one for the unsegmented model, and one for each segment). In contrast stratified/importance-sampling is more flexible by virtue of the scenario weighting (Equations 16 and 18). This enables sharing of Monte-Carlo scenarios between the unsegmented model and the segments, and is an important advantage over stratified-sampling.

In our onshore application a single Monte-Carlo sample is created by setting $r_{*}(e)$ equal to the logic-tree mean model $\bar{r}(e)$. The chance of sampling each scenario in magnitude-bin $M_{w, b}$ (Equation 16) is thus defined as:

$$
w_{b, i}^{S I S}(e)=\frac{\mathcal{I}(e) \bar{r}(e)}{\sum_{e \in E_{b}} \mathcal{I}(e) \bar{r}(e)}
$$

which does not change when varying $i \in I$. With this approach the reasoning behind Equations 17-25 remains valid, whether applied to different segment models (Tonga, Kermadec, or Hikurangi) or the unsegmented model. Therefore each of these four models can use the same set of 1200 scenarios. This offers a potentially large improvement over stratified-sampling, where the need to partition the 1200 scenarios among the 4 source models would result in lower sample sizes on each.

In practice the benefit of scenario sharing depends on the extent to which different source models assign non-zero probabilities to the same scenarios, in addition to how $\mathcal{I}(e)$ is defined. Thus the performance should be checked in each application at offshore sites, prior to expensive inundation simulation. Here this is deferred to Section 3.5 where we also assess the effect of non-uniform magnitude-bin sampling.

\subsection{Improving the efficiency: Non-uniform sampling of magnitude-bins}

This section develops an approach to determine the number of samples in each magnitude-bin $N\left(M_{w, b}\right)$. Both stratified and stratified/importance-sampling require fixing $N\left(M_{w, b}\right)$ prior to sampling, and in practice uniform $N\left(M_{w, b}\right)$ are often used (e.g. De Risi \& Goda, 2017; Williamson et al., 2020; Zamora et al., 2021). However the computational effort is generally related to the total number of scenarios $N_{t o t}$ :

$$
N_{\text {tot }}=\sum_{M_{w, b} \in \text { Magnitude bins }} N\left(M_{w, b}\right)
$$

which determines how many high-resolution tsunami simulations are required. Below we develop a strategy to select non-uniform $N\left(M_{w, b}\right)$ values that reduce the Monte-Carlo errors while keeping $N_{t o t}$ constant. The approach is found to be beneficial, especially for relatively rare large tsunamis.

As a starting point it is shown that, prior to sampling, optimal values of $N\left(M_{w, b}\right)$ can be computed at any offshore site using the offshore PTHA, given a specific threshold $Q^{T}$ and scenario-frequency model $i \in I$. The solution is optimal in the sense that it minimises the variance of the Monte-Carlo exceedance-rate estimates. Similar problems and solution strategies are common in sampling theory (e.g. Thompson, 2012).

For both stratified and stratified/importance-sampling, the analytical variance of the Monte-Carlo exceedancerate estimate is of the form (Equations 12 and 23):

$$
\sum_{M_{w, b} \in \text { Magnitude bins }} \frac{\alpha_{i}\left(M_{w, b} \mid Q^{T}\right)}{N\left(M_{w, b}\right)}
$$

where the $\alpha_{i}\left(M_{w, b} \mid Q^{T}\right)$ are obtained by removing the factor $1 / N\left(M_{w, b}\right)$ from the within-magnitude-bin variance, i.e. using Equation 9 for stratified-sampling:

$$
\alpha_{i}\left(M_{w, b} \mid Q^{T}\right)=\left(\lambda_{i}\left(M_{w, b}\right)\right)^{2} p_{b, i, T}\left(1-p_{b, i, T}\right)
$$

and Equation 19 for stratified/importance-sampling:

$$
\alpha_{i}\left(M_{w, b} \mid Q^{T}\right)=\left(\lambda_{i}\left(M_{w, b}\right)\right)^{2}\left(\sum_{e \in E_{b}}\left(\left[\mathbb{1}_{\left(Q(e)>Q^{T}\right)} \phi_{b, i}^{S I S}(e)-p_{b, i, T}\right]^{2} w_{b, i}^{S I S}(e)\right)\right)
$$

In either case the values of $N\left(M_{w, b}\right)$ that minimise the variance (Equation 28) subject to the constraint of fixed $N_{t o t}$ (Equation 27) can be derived using Lagrange multipliers, and are denoted $N_{i}\left(M_{w, b} \mid Q^{T}\right)$ :

$$
N_{i}\left(M_{w, b} \mid Q^{T}\right)=N_{t o t} \sqrt{\alpha_{i}\left(M_{w, b} \mid Q^{T}\right)} /\left(\sum_{M_{w, b} \in \text { Magnitude bins }} \sqrt{\alpha_{i}\left(M_{w, b} \mid Q^{T}\right)}\right)
$$




\section{Stratified sampling}
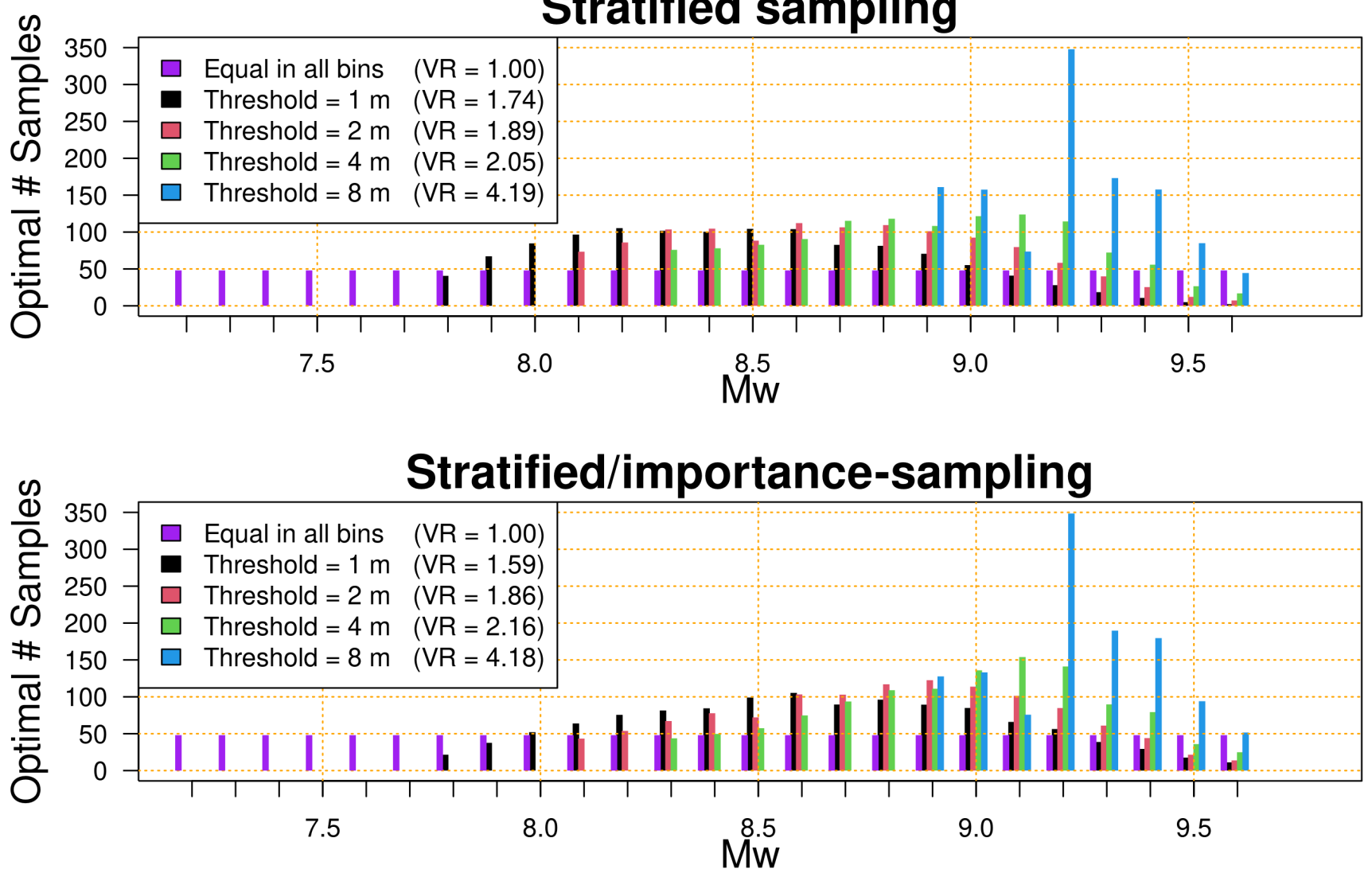

Figure 5: Optimal number of samples in each magnitude-bin for stratified-sampling (top) and stratified/importancesampling (bottom), computed with Equation 31 for a range of thresholds $Q^{T}$ at Site P. The logic-tree mean model $\bar{r}(e)$ was used to specify scenario-frequencies, and $N_{t o t}=1200$. VR gives the relative reduction in the Monte-Carlo variance at each threshold due only to use of non-uniform $N\left(M_{w, b}\right)$. In the top panel VR compares stratified-sampling with uniform versus non-uniform $N\left(M_{w, b}\right)$. In the bottom panel VR compares stratified/importance-sampling with uniform versus non-uniform $N\left(M_{w, b}\right)$.

For practical applications Equation 31 should be rounded to give integer solutions in each magnitude-bin.

The optimal sampling effort (Equation 31) is subject to important limitations; it can only be computed where the offshore PTHA is accurate (i.e. not at coastal sites) and is not necessarily robust for alternative scenario-frequency models $i \in I$, alternative offshore sites, or alternative thresholds. However we suggest that good sampling strategies (albeit non-optimal) can be inferred by inspecting optimal solutions at offshore sites near the coastal site of interest, combined with judgement about the tsunami wave-heights of most significance to the application.

At Site $\mathrm{P}$, by inspection it is clear that the optimal non-uniform sampling effort varies substantially with the selected threshold $Q^{T}$ (Figure 5). Both stratified and stratified/importance-sampling show a similar trend: at higher thresholds it is better to concentrate sampling on higher magnitudes. The efficiency improvement can be computed from the ratio of the variances when using uniform and non-uniform $N\left(M_{w, b}\right)$, while keeping other aspects of the sampling scheme fixed. This is quantified with VR in Figure 5, where variances were computed with Equation 28. We stress these VR values show the extra reduction of variance due to non-uniform versus uniform $N\left(M_{w, b}\right)$, not the combined accuracy of each sampling technique. Stratified/importance-sampling will have much lower variance overall for reasons discussed above (Section 3.3.1), but here we only consider the improvement due to non-uniform $N\left(M_{w, b}\right)$. For the thresholds reported in Figure 5, VR shows that non-uniform sampling reduces the variance by a factor of $1.6-4.2$, for both stratified and stratified/importance sampling. There is greater potential for variance reduction at higher thresholds, because low-magnitude earthquakes are unlikely to generate large waves.

The full variance reductions in Figure 5 cannot practically be obtained because only one set of $N\left(M_{w, b}\right)$ can be selected; the sampling effort cannot be tailored to every threshold and scenario-frequency model. Therefore, we suggest a compromise approach to determining non-uniform $N\left(M_{w, b}\right)$, informed by the optimal solutions and 
site-specific judgement:

1. For robustness $1 / 4$ of the scenarios are uniformly distributed among all magnitude-bins. This guarantees the Monte-Carlo standard-deviation for any site or scenario-frequency model will never exceed twice the value that would have been obtained using uniform $N\left(M_{w, b}\right)$.

2. The remaining $3 / 4$ of scenarios are assigned non-uniformly, in proportion to the optimal $N\left(M_{w, b}\right)$ averaged over stage-thresholds of 1, 2, 4, and $8 \mathrm{~m}$ at the same Site $\mathrm{P}$ used to define the scenario importance (Figure 5). These thresholds are expected to vary between applications. Herein they were chosen after some experimentation, because they cover mean exceedance-rates around 1/500 - 1/10000 which are likely to be important in our application, and lead to reasonable variance reductions.

For these calculations we used the logic-tree mean scenario rate model $\bar{r}(e)$, restricted to thrust scenarios on the Kermadec-Tonga source. While other scenario-frequency models in $I$ could be used, the mean model is a reasonable focus for optimisation, as it is often used for inundation mapping at nominal return periods.

The resulting non-uniform $N\left(M_{w, b}\right)$ leads to more sampling at $M_{w} 8.3-9.4$, and less sampling at low and very high magnitudes (Figure 6). The associated variance-reductions are necessarily less than the optimal VR for the same threshold (compare VR values in Figures 6 and 5). However they are still equivalent to using 1.1-2.0 times more scenarios, relative to using uniform $N\left(M_{w, b}\right)$ (Figure 6). The benefit of non-uniform sampling increases at higher threshold values, which is desirable because larger tsunamis tend to have greater Monte-Carlo errors (Figures 2 and 3). In practice we should also check the performance at sites other than Site P; this is the focus of Section 3.5.

\subsection{Testing the sampling scheme at offshore sites prior to inundation simulation}

Before selecting a sampling scheme and running expensive inundation computations we recommend testing the approach at nearby offshore sites, using the offshore PTHA. This has minimal computational cost and allows any problems with the sampling scheme to be identified and fixed prior to expensive tsunami simulation. Here the new Monte-Carlo scheme is compared with regular stratified-sampling with uniform $N\left(M_{w, b}\right)$, which represents the standard approach. In both cases $N_{t o t}=1200$. The new scheme combines stratified/importance-sampling based on Site $\mathrm{P}$ with non-uniform $N\left(M_{w, b}\right)$ (Figure 6). The same Monte-Carlo sample is re-used for both segmented and unsegmented source models, as explained in Section 3.3.2. Section 4 employs the same sampling scheme for probabilistic inundation computations in Tongatapu.

Figure 7 depicts 95\% confidence-intervals for the logic-tree mean hazard curves at the same sites used in Figure 4. The relative variance reductions at $Q^{T}=2,4 \mathrm{~m}$ compare the variance of the new scheme with the variance of the standard approach (Figure 7). They show the new scheme improves the efficiency by a factor of 4.5-10.8 near Tongatapu, with all sites showing a greater efficiency improvement for larger waves. At distant site NZ there is a small efficiency reduction, reflecting that stratified/importance sampling can reduce the efficiency far from Site $\mathrm{P}$ (which was used to define the scenario importance $\mathcal{I}(e)$ and non-uniform $N\left(M_{w, b}\right)$ values). But near Tongatapu the improvements are substantial.

To highlight the benefits of re-using scenarios for epistemic uncertainty calculations, the calculations were repeated with $100 \%$ weight on the unsegmented source-zone representation (Figure 8). The stratified/importance sampling strategy was unchanged, matching the approach used in practice (Section 4). Epistemic uncertainty calculations via stratified-sampling require that the 1200 scenarios are shared among the four source models (unsegmented and three segments; Section 3.2.2). Thus the stratified-sampling results in Figure 8 assume only 600 of the 1200 Monte-Carlo scenarios are used to sample the unsegmented model. This is a fair comparison because in practice, the other 600 Monte-Carlo scenarios would be used to sample the segmented models, which are assigned $50 \%$ weight in PTHA18.

Figure 8 shows that stratified/importance-sampling leads to even greater efficiency improvements for the unsegmented model (Figure 8), as compared to the improvements observed for the logic-tree-mean model (Figure 7). Sites near Tongatapu exhibit variance reductions of a factor 7.7-18.4 at thresholds $Q^{T}=2,4 \mathrm{~m}$, with greater improvements for larger tsunamis. In this case even distant site NZ exhibits a small efficiency improvement. This highlights the benefit of Monte-Carlo sample re-use when calculating epistemic uncertainties.

The results in Figures 7 and 8 confirm that the sampling scheme is likely to perform well near Tongatapu. This provides confidence in the applicability of our stratified/importance-sampling approach. The following section applies the technique to onshore hazard assessment. 


\section{Selected non-uniform sampling effort and extra variance-reduction}

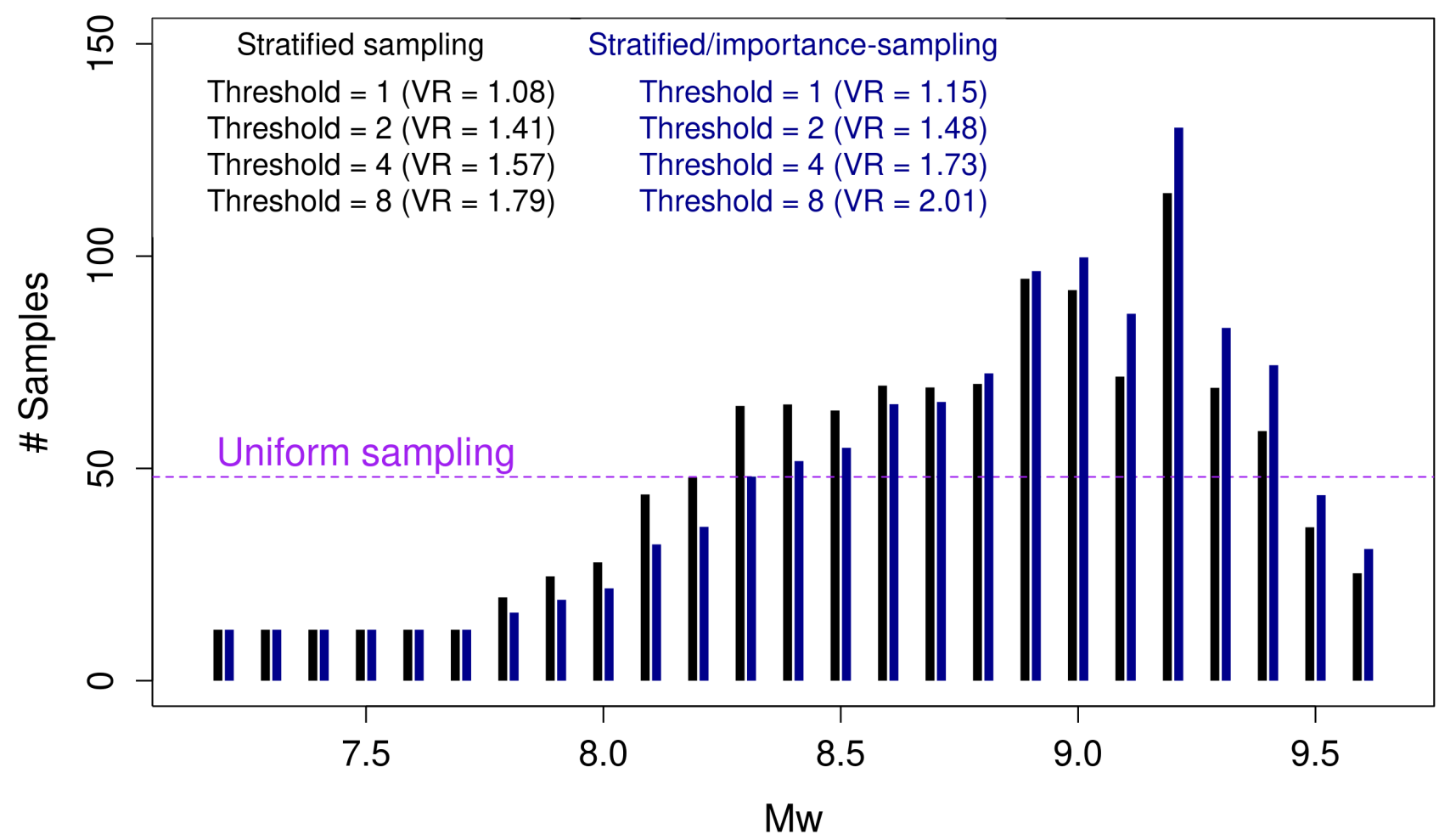

Figure 6: The selected number of samples in each magnitude-bin for both stratified-sampling and stratified/importance-sampling. This is a compromise between the optimal solutions for different $Q^{T}$ values. VR gives the relative reduction in the Monte-Carlo variance at the specified threshold due only to use of non-uniform $N\left(M_{w, b}\right)$. The black VR values compare stratified-sampling using uniform versus non-uniform $N\left(M_{w, b}\right)$, while the blue VR values compare stratified/importance-sampling using uniform versus non-uniform $N\left(M_{w, b}\right)$. The results apply to the offshore Site $\mathrm{P}$ that was used to define the scenario importance, with exceedance-rates evaluated using the logic-tree mean scenario-frequency model, and $N_{t o t}=1200$. 

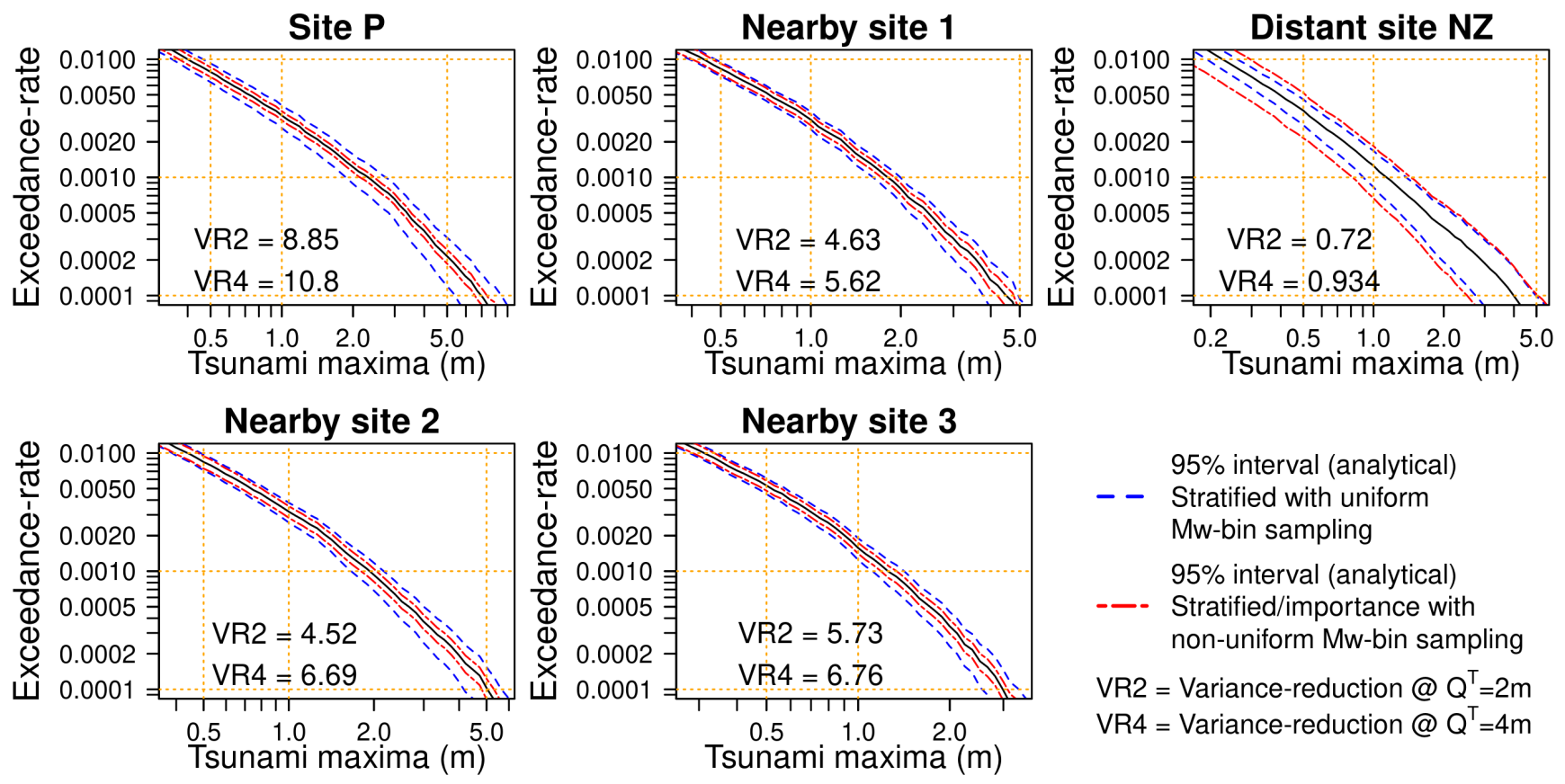

Figure 7: Monte-Carlo variability in the logic-tree mean exceedance-rate curves at offshore sites. Regular stratifiedsampling with uniform $N\left(M_{w, b}\right)$ is compared with the new scheme (stratified/importance-sampling with non-uniform $N\left(M_{w, b}\right)$, details matching Figure 6). The 95\% intervals and relative variance reductions were derived analytically (Equations 12 and 23), assuming a normal distribution for the confidence-intervals. Note the VR2 and VR4 values show the efficiency improvements of stratified/importance-sampling with non-uniform $N\left(M_{w, b}\right)$, relative to stratifiedsampling with uniform $N\left(M_{w, b}\right)$.
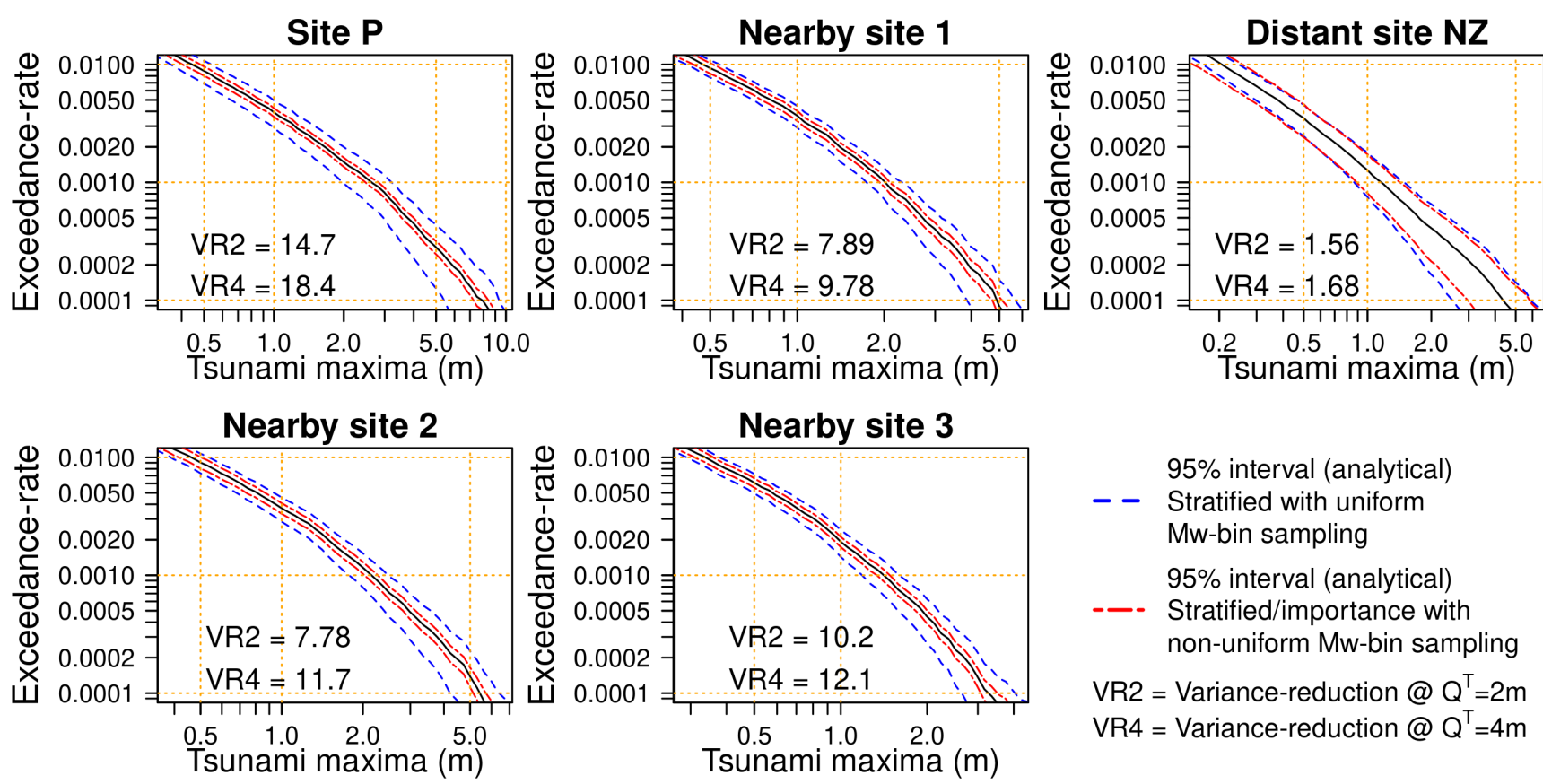

Figure 8: Monte-Carlo variability in the exceedance-rate curves at offshore sites with $100 \%$ weight on the unsegmented scenario-frequency model. Other details are the same as Figure 7 except stratified-sampling uses 600 scenarios (assuming the other half would be used to sample the segmented models). Stratified/importance-sampling uses 1200 scenarios with the same sampling approach as Figure 7. 


\section{APPLICATION TO PROBABILISTIC INUNDATION COMPUTA- TION}

\subsection{Tsunami scenarios and inundation computation}

A single Monte-Carlo sample with 1200 scenarios was constructed using stratified/importance-sampling with nonuniform $N\left(M_{w, b}\right)$ based on Site P, exactly matching the approach in Section 3.5. Although we only sampled thrust scenarios from the Kermadec-Tonga trench, a more comprehensive hazard assessment could include scenarios from other PTHA18 source-zones. This would increase the frequency of smaller tsunamis, and could be done by repeating the computations herein for multiple source-zones. Alternatively, multiple source-zones could be sampled at once by combining all in the set of scenarios E, using the same equations presented in Sections 3.3 and 3.4.

For all randomly sampled scenarios, the tsunami was simulated from source through to inundation with the shallow water equations using ocean-surface perturbations from PTHA18 (Davies \& Griffin, 2018). They were applied instantaneously to both the initial ocean surface, and the elevation. Scenarios were run with an initial (preearthquake) sea-level of $0 \mathrm{~m}$, and separately $0.8 \mathrm{~m}$, to approximate the mean-sea-level and monthly tidal maxima at Tongatapu. This gives some insight into the importance of tides; a more comprehensive treatment of tides is beyond the scope of this study (Lane et al., 2012; Adams et al., 2015; Wilson \& Power, 2020; González et al., 2021).

The model domain (Figure 9) uses three levels of two-way grid nesting. The outer domain has $\simeq 1850 \mathrm{~m}$ cell-size, while high resolution areas around Tongatapu have $\simeq 7.5 \mathrm{~m}$ cell size. Elevation data was derived from a mixture of lidar, gridded bathymetric surveys near Tongatapu (Damlamian et al., 2013), and global-scale data derived from GA250 (Whiteway, 2009) and GEBCO-2014 Grid (version 20150318).

Tsunamis were simulated using the open-source SWALS code, which provides a number of explicit shallow water solvers including the linear solver used for PTHA18 (Davies \& Griffin, 2018, 2020). This code has a test-suite of more than 20 analytical, laboratory or field problems, including well known tsunami problems from NTHMP (2012) (excluding landslides) and other recent NTHMP problems (Park et al., 2013; Lynett et al., 2017; Macías et al., 2020; Gao et al., 2020). The model setup herein mirrors that used by Davies et al. (2020) to compare modelled and observed tsunamis at 16 Australian tide-gauges. On all nested grids a second-order finite-volume scheme is used to solve the full nonlinear shallow water equations with uniform Manning-friction of 0.03 (details similar to Davies \& Roberts, 2015). On the outer domain the linear shallow water equations are combined with Manning-friction and solved using a classical scheme (Goto \& Ogawa, 1997), with details matching Davies et al. (2020).

To check the model performance, a number of convergence tests were conducted by factor-of-two coarsening of the model resolution. This had a small impact on the hydrodynamic results, suggesting the model resolution is fine enough. We also simulated five historic earthquake-tsunamis measured on the Nuku'alofa tide-gauge (Figure 9). These resulted from earthquakes in Tonga 2006/05/03 $\left(M_{w} 8.0\right)$, Tonga 2009/09/29 $\left(M_{w} 8.1\right)$, Chile 2010/02/27 $\left(M_{w} 8.8\right)$, Tohoku 2011/03/11 $\left(M_{w} 9.1\right)$, and Chile 2015/09/16 $\left(M_{w} 8.3\right)$. For each historic event a similar tsunami scenario was selected from among PTHA18 scenarios that fit DART buoy observations relatively well (as identified in Davies, 2019). To simulate the Tohoku and Chile events the outer domain was extended to cover the Pacific Ocean and it was evolved for 24 hours. For the Tonga events the model was evolved for 5 hours with an outer-domain matching Figure 9, identical to that used to simulate the random scenarios.

For each historical event, the model gives a reasonable representation of the tsunami size and arrival time (Figure 9). Detailed investigations of these historical events is outside the scope of our study. However, improved agreement between the modelled and observed waveforms could likely be obtained via refinement of the source models (Bosserelle et al., 2020) and inclusion of loading and dispersion in the shallow water model (Allgeyer \& Cummins, 2014; Watada et al., 2014; Baba et al., 2017).

\subsection{Compatibility with the offshore PTHA}

To check the results we compared the offshore PTHA18 with the high-resolution Monte-Calro results at Site P (Figure 10). This is an important test because, in principle, the exceedance-rate curves should agree well at deep water sites far from the coast, where the linear assumptions underling the offshore PTHA are reasonable (Gibbons et al., 2020; Tonini et al., 2021). Significant differences would warrant investigation because they could indicate implementation errors, or the use of too small a Monte-Carlo sample with the high-resolution model. Conversely, they might simply reflect differences in the hydrodynamic models and elevation data used in both studies (Gibbons et al., 2020). For example, compared to our high-resolution model (Section 4.1) the PTHA18 hydrodynamic model is much simpler; it solves the frictionless linear shallow water equations at a globally-uniform 1 arc-minute resolution for 36 hours duration (Davies \& Griffin, 2020). 

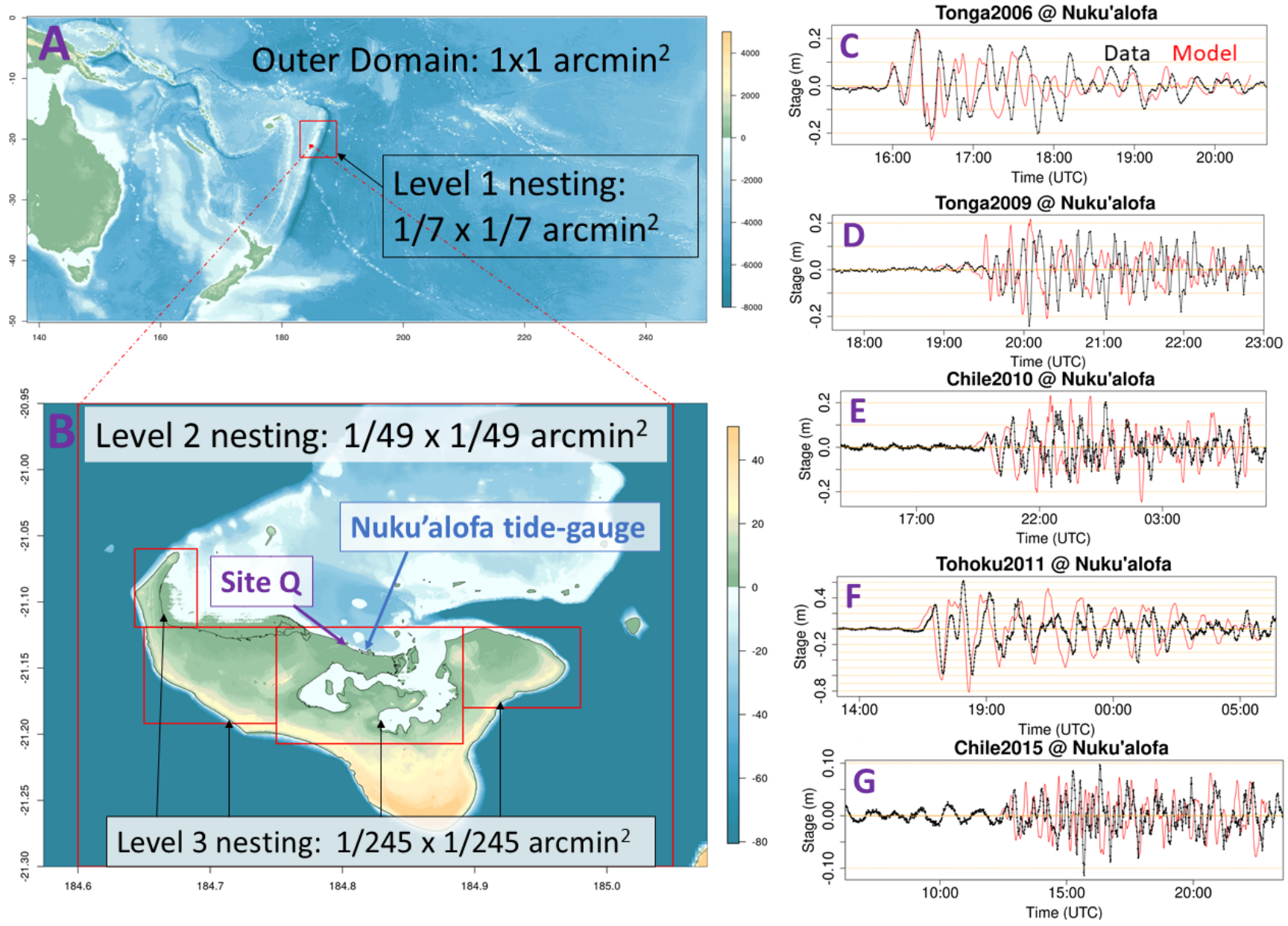

Figure 9: Model structure and comparison of models with historic tsunamis at Nuku'alofa tide-gauge. A) Outer domain used to simulate all scenarios originating from the Kermadec-Tonga source. To simulate historic tsunamis from Chile and Tohoku, the outer domain was extended to include the tsunami source. B) Model design near Tongatapu, with locations mentioned in the text. Black solid line is the OpenStreetMap coastline (C) OpenStreetMap contributors). The highest resolution domains cover low-lying built-up areas of northern Tongatapu. $C$ - $G$ ) Comparison of historic tsunamis and models at Nuku'alofa tide-gauge. All model results are derived from PTHA18 scenarios with earthquake magnitude and location similar to the historical event (Davies, 2019). 
A) Monte-Carlo uncertainties

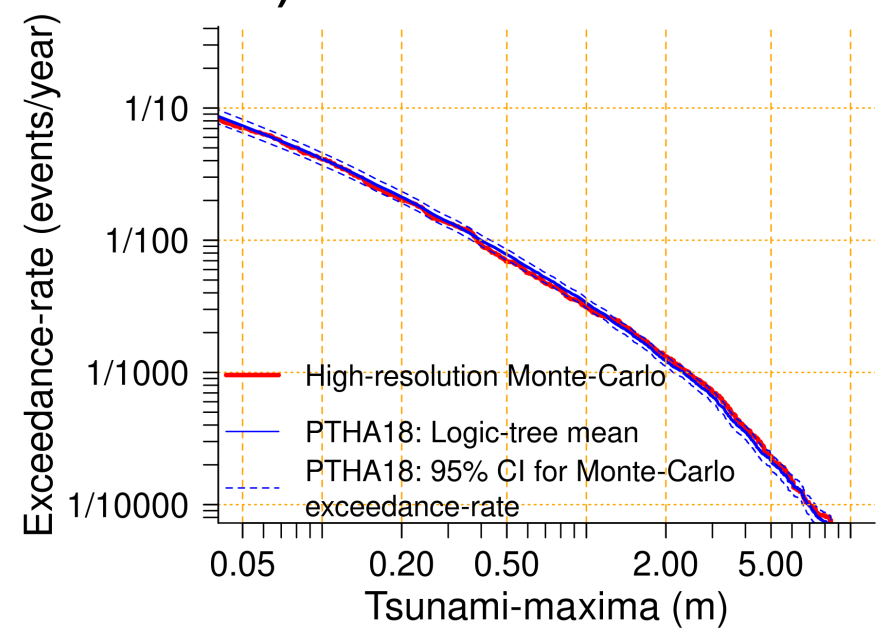

B) Epistemic uncertainties

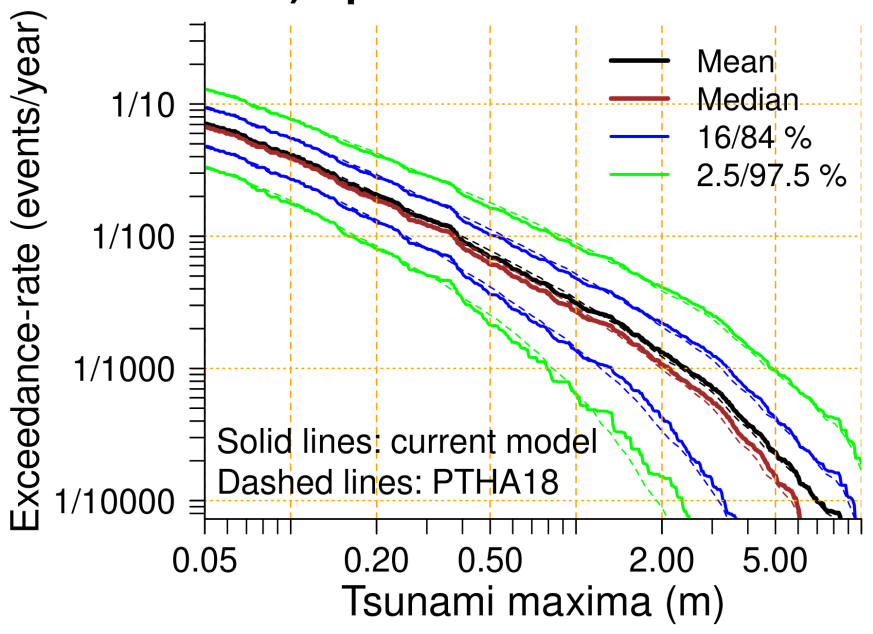

Figure 10: Consistency of the original PTHA18 and the Monte-Carlo results at offshore Site P, using Kermadec-Tonga scenarios and a background sea-level of $0 \mathrm{~m}$. A) The maximum-stage exceedance-rate curve for the logic-tree mean model, as computed in PTHA18, and with the high-resolution Monte-Carlo model. The $95 \%$ confidence-intervals were derived analytically from the PTHA18, assuming Monte-Carlo errors for the sampling scheme are normally distributed with the mean and variance derived from Equations 1 and 23. B) Comparison of epistemic uncertainties in the tsunami-maxima exceedance-rates from the PTHA18 (dashed lines) and the Monte-Carlo results herein (solid lines).

Despite differences in their hydrodynamics, Figure 10 shows there is good agreement between the high-resolution Monte-Carlo results and the PTHA18 results at Site P. For the logic-tree mean exceedance-rate curve, the highresolution Monte-Carlo results are largely within the 95\% analytical Monte-Carlo confidence-interval derived from the offshore PTHA (Figure 10A). There is also good agreement between percentiles of epistemic uncertainties as derived in both studies (Figure 10B). These epistemic uncertainty calculations follow the methodology of PTHA18, which employs a conservative model of dependence between the Tonga, Kermadec and Hikurangi segments (details in Davies \& Griffin, 2020). The agreement in Figure 10 gives confidence that the high-resolution Monte-Carlo calculations have been correctly implemented.

Greater differences were reported in a similar test by Gibbons et al. (2020) using the TSUMAPS-NEAM offshore PTHA and a set of more than 30,000 high-resolution scenarios (selected with non-random sampling). This may reflect that their comparison site was located in relatively shallow water (50 m deep) about $6 \mathrm{~km}$ offshore. The effects of nonlinear hydrodynamics and high-resolution bathymetry are likely to be more pronounced at such a site, as compared to Site $\mathrm{P}$ which is $18 \mathrm{~km}$ offshore Tongatapu in $800 \mathrm{~m}$ depth. Because nearshore and shallow-water effects make it harder to notice problematic differences between the offshore and nearshore PTHAs (e.g. caused by implementation errors or insufficient sampling), we recommend testing at a deep-water site far from the coast, if available in the offshore PTHA.

\subsection{Probabilistic Inundation: Monte-Carlo Accuracy and Epistemic Uncertainty}

To study the Monte-Carlo accuracy onshore we consider 'Site Q' in Tongatapu, which is inland about $150 \mathrm{~m}$ from the shoreline (location in Figure 9B). Similar analyses can be applied to any onshore site of interest. Depth exceedancerates were computed for the logic-tree mean model, and 95\% confidence-intervals for the exceedance-rate in Equation 1 were computed with Equation 25 (Figure 11A). Results with 100\% weight on the unsegmented or union-of-segments source representations are also shown (Figure 11A). The $95 \%$ exceedance-rate intervals range over $\simeq 9 \%$ of the estimated exceedance-rate at $1 / 500$, growing slowly to $\simeq 11 \%$ at $1 / 2500$ and $\simeq 16 \%$ at $1 / 10000$. These numbers are similar for all scenario-frequency models in Figure 11A. If the high-resolution Monte-Carlo calculations were repeated many times with different random scenarios, then the exceedance-rate (Equation 1) for each source-model would be within these intervals approximately $95 \%$ of the time.

For a fixed exceedance-rate, Monte-Carlo uncertainties in the depth can also be inferred from horizontal transects along Figure 11A. For the logic-tree mean model, the upper limit of the $95 \%$ interval differs from the computed 

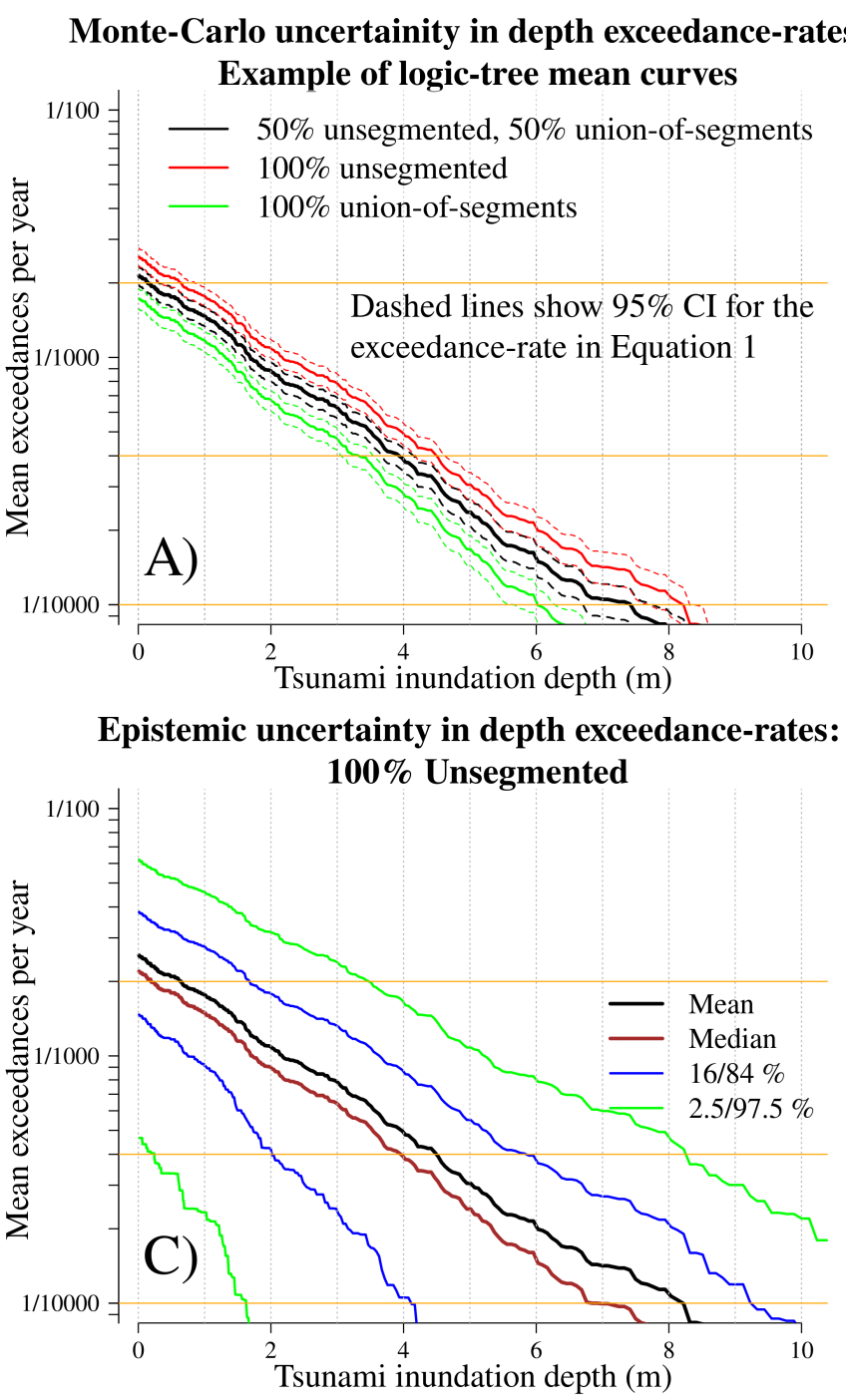
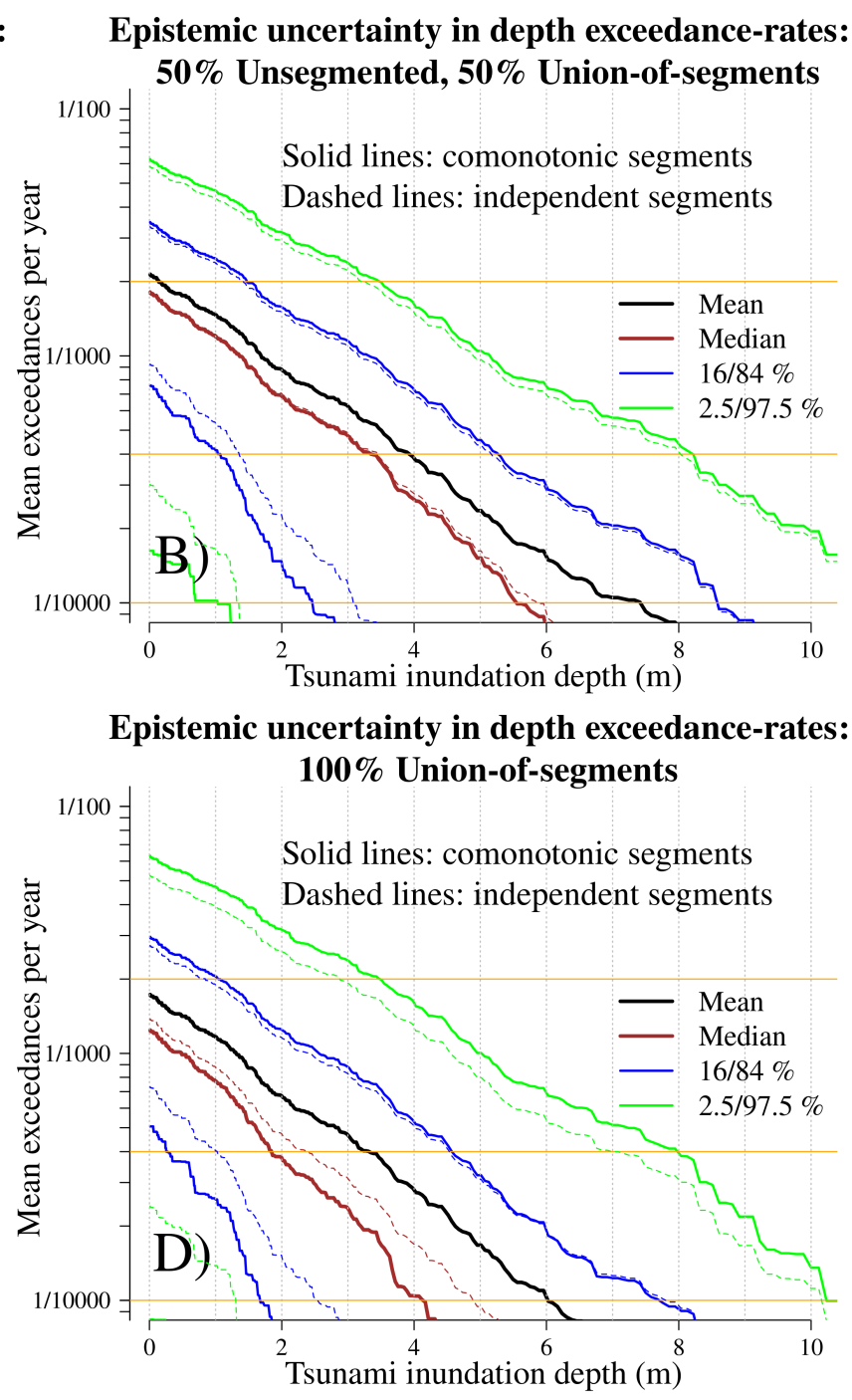

Figure 11: Monte-Carlo and epistemic uncertainties in inundation depth exceedance-rates at Site Q (lon,lat $=$ 184.8017,-21.13305). The background sea-level is $0 \mathrm{~m}$ and only Kermadec-Tonga trench thrust scenarios are included. A) Logic-tree-mean curves for 3 different scenario-frequency representations, with 95\% confidence-intervals (computed via Equation 25) for the exceedance-rate in Equation 1. B) Epistemic uncertainties in the inundationdepth exceedance-rates. Percentiles vary slightly if the segments are treated as comonotonic (solid lines) or independent (dashed lines). C) Same as panel B but with $100 \%$ weight on the unsegmented source representation. D) Same as panel $B$ but with $100 \%$ weight on the union-of-segments source representation. 
mean by $+16 \mathrm{~cm}$ (at $1 / 500$ exceedance-rate), $+22 \mathrm{~cm}(1 / 2500)$, and $+33 \mathrm{~cm}(1 / 10000)$. Based on the offshore results (Section 3.5) we anticipate these intervals would be $\simeq 2-4.3$ times wider if the calculations had used regular stratified-sampling with uniform $N\left(M_{w, b}\right)$ and the same computational effort; in that case it would be difficult to distinguish the different source representations in Figure 11A.

The Monte-Carlo confidence-intervals in Figure 11A reflect the expected error given a limited number of random scenarios $\left(N_{t o t}=1200\right)$ and could be reduced using a larger Monte-Carlo sample. However, they are already small compared to the epistemic uncertainties (Figure 11B) which reflect our uncertain knowledge of earthquake magnitude-frequency relations on the Kermadec-Tonga trench (Figure 1C-F). In tsunami risk management practice the $84^{\text {th }}$ percentile depth is sometimes used in preference to the mean (Leonard et al., 2008; MCDEM, 2016; Tonini et al., 2021); in Figure 11B the $84^{\text {th }}$ percentile curve exceeds the mean curve by a depth of $1-2 \mathrm{~m}$ for a given exceedance-rate. That difference is substantially greater than the Monte-Carlo uncertainties (Figure 11A) suggesting limited practical benefit to using a larger $N_{t o t}$ at this site, given our novel sampling scheme.

Some conceptual subtleties arise in the epistemic uncertainty calculations (Figure 11B-D), depending on how the segmented models are combined. To calculate epistemic uncertainties the Monte-Carlo exceedance-rate estimates (Equation 22) are first computed for all logic-tree scenario-frequency models (corresponding to individual grey curves in Figure 1C-F). Each scenario-frequency model has a Bayesian posterior weight determined as in Davies \& Griffin (2020), and for any depth threshold these combine to give a distribution of exceedance-rates. There is one such distribution for the unsegmented model, and one for each of the three segments. Percentiles can be computed to summarise these distributions for any depth threshold, as depicted for the unsegmented model in Figure 11C. However, the union-of-segments case (Figure 11D) is more complex because three segments lead to three distributions which must be combined, and the distribution of their union is affected by any dependence between the segments (although the mean is unaffected). Davies \& Griffin (2020) argued that dependence in epistemic-uncertainties should be used to reflect the possibility that similar physical processes control maximum earthquake magnitudes, coupling, etc. This increases the likelihood that relatively high (or low) epistemic-uncertainty percentiles are true on all segments simultaneously. PTHA18 conservatively accounts for this by assuming comonotonic dependence between segments, i.e. if the $84^{\text {th }}$ percentile exceedance-rate is true on one segment, it is true on all segments simultaneously (and similarly for other percentiles, Deelstra et al., 2009). While the comonotonic approach is also used herein, to better understand its effect we repeated the calculations assuming independent segments (Figure 11D). The inter-segment dependence treatment also affects the full model (Figure 11B) because it is a 50:50 sum of the unsegmented and union-of-segments probability densities. However at this site the effects of comonotonic dependence vs independence of segments are small for higher percentiles, although significant for lower percentiles (Figures 11B and D). This is because higher percentiles are predominantly affected by one segment (Tonga) which is near-field; the other two segments are less likely to generate large waves at Tongatapu, so contribute much less to high-hazard percentiles irrespective of inter-segment dependence. While the dependence treatment does not have a major influence on epistemic uncertainties at this site, the impacts could be greater elsewhere if multiple segments contributed more equally to the hazard.

The spatial distribution of depths with exceedance-rate of $1 / 2475$ (equivalent to a $2 \%$ chance of exceedance in 50 years) were computed for the logic-tree mean curve as well as the $84^{\text {th }}$ and $16^{\text {th }}$ percentiles (Figure 12AC). This was implemented by repeating site-specific calculations like in Figure 11 at each pixel, using root-finding (Brent, 1973) to find $Q^{T}$ values corresponding to the desired exceedance-rate. The difference between the $16^{\text {th }}$ and $84^{\text {th }}$ percentile results (Figure 12B-C) highlight the large epistemic uncertainties in inundation depths, which stem from large uncertainties in PTHA18's magnitude-frequency relations for the Kermadec-Tonga trench (Figure 1C-F). Low-lying areas along the north coast are inundated in all cases, while elsewhere, the steep coastal topography of Tongatapu limits the inundation extent.

The results in Figure 12A-C assume a background sea-level equal to mean-sea-level, whereas in practice tides at Tongatapu have a typical monthly maxima of $0.8 \mathrm{~m}$. If the tsunami maxima were to coincide with such a high-tide then the logic-tree mean depths increase (compare Figures 12D and A) but are still smaller than the $84^{\text {th }}$ percentile depths with a background sea-level of $0 \mathrm{~m}$ (Figure 12B). Although not undertaken herein, the results suggest that while tidal aleatory uncertainties are significant at Tongatapu, they are less influential for understanding tsunami hazards than epistemic uncertainties in source frequencies. However by 2100 sea-levels at Nuku'alofa are projected to rise by approximately $0.8 \mathrm{~m}$ due to climate change (Fox-Kemper et al., 2021), so in future we expect an increasing chance of relatively high sea-levels exacerbating tsunami inundation (Li et al., 2018; Sepúlveda et al., 2021). 

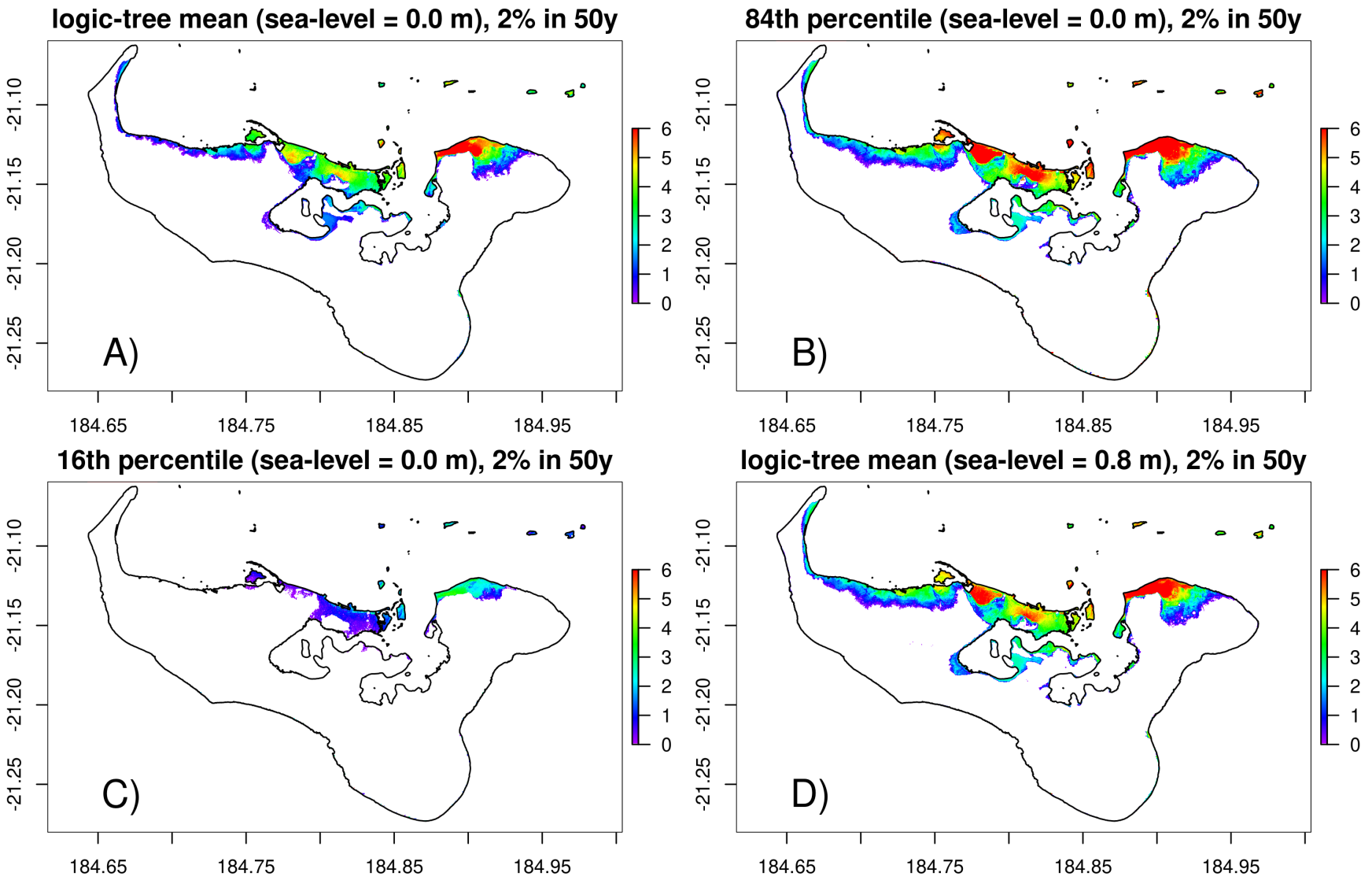

Figure 12: Inundation depth with a $2 \%$ chance of exceedance in 50 years. Solid line is the OpenStreetMap coastline (C) OpenStreetMap contributors). A) Logic-tree mean with background sea-level of $0 \mathrm{~m}$. B) $84^{\text {th }}$ percentile of the epistemic uncertainty with a background sea-level of $0 \mathrm{~m}$. C) $16^{\text {th }}$ percentile of the epistemic uncertainty with a background sea-level of $0 \mathrm{~m}$. D) Logic-tree mean with a background sea-level of $0.8 \mathrm{~m}$. 


\section{SUMMARY AND CONCLUSIONS}

Offshore PTHAs are increasingly available globally and provide a foundation for high-resolution onshore PTHAs, which are useful to guide tsunami risk-management. To translate the offshore hazard to an onshore site of interest, ideally high-resolution tsunami simulations would be conducted for every scenario in the offshore PTHA, but this is usually computationally prohibitive. Monte-Carlo sampling offers an attractive alternative that converges to this ideal solution, and can have acceptable accuracy at much lower computational cost. Theoretical results enable the performance of all sampling schemes considered herein to be understood at offshore sites, where the PTHA is valid, prior to sampling. This enables proposed Monte-Carlo sampling schemes to be checked, and perhaps further optimised, prior to expensive tsunami computation. Furthermore, once the inundation computations are available, the Monte-Carlo error at coastal sites can be estimated from the single Monte-Carlo sample, and offshore results can be cross-checked with the offshore PTHA. In combination these techniques facilitate the control of Monte-Carlo errors when translating from offshore to onshore PTHA.

The key novel contributions of this paper involve using the offshore PTHA to improve the Monte-Carlo efficiency near a site of interest. Stratified/importance-sampling enables better sampling of scenarios that are likely to be important according to user-defined criteria (based on offshore tsunami maxima herein). Compared to stratifiedsampling this can substantially reduce Monte-Carlo errors for a given computational cost, both because it better represents important scenarios, and because it facilitates greater re-use of the Monte-Carlo sample in epistemic uncertainty calculations. Further efficiency improvements were obtained via non-uniform sampling of magnitudebins, informed by theoretically optimal solutions near the site of interest. The latter technique can be combined with both stratified and stratified/importance-sampling. In our application, the combination of these techniques led to efficiency improvements near Tongatapu, equivalent to using $\simeq 4-18$ times more scenarios with uniform stratified-sampling. These efficiency improvements vary from site to site but are consistently greater for rare return periods, and quantification of epistemic-uncertainties. Because onshore PTHA is often limited by the computational cost, this will help enable PTHA to be used for a greater range of onshore hazard applications.

In future it would be interesting to try combining the techniques here with fast approximate inundation computation (e.g. de Baar \& Roberts, 2017; Guillas et al., 2018; Volpe et al., 2019; Williamson et al., 2020; Fukutani et al., 2021; Makinoshima et al., 2021). This will involve some trade-offs that remain to be fully explored; for example, while Monte-Carlo errors could be reduced using many approximate inundation scenarios, the latter may introduce systematic errors that offset the benefit. In such cases the approximate inundation may still be of value to guide the scenario importance definition. There is also scope to extend stratified/importance-sampling to more complex situations. For example, dynamic tides might be treated by considering the tidal-phase as a random variable and integrating it into the user-defined scenario importance, to reflect that greater inundation is expected near high-tides. The techniques could also be extended to other kinds of tsunami sources (e.g. landslides), if useful definitions of the scenario importance can be invented. Further consideration should also be given to the treatment of multiple sourcezones, as our method offers two approaches to this. One is to combine multiple sources into the set of scenarios $E$ prior to sampling; the other is to sample each source-zone separately with a chosen number of scenarios. The former approach will cause the number of scenarios on each source to be randomly determined, with better representation of source-zones that often produce important scenarios, but potentially limited representation of others. The latter approach gives the modeller control over the representation of each source-zone, and would also permit the use of varying scenario importance definitions for each.

Finally, we stress key limitations to this work. Although our techniques are general, the probabilistic inundation hazard results presented here only reflect PTHA18 thrust scenarios on the Kermadec-Tonga source zone, and neglect outer-rise earthquakes, more distant earthquake sources, and other sources such as landslides or volcanoes. The model does not account for dynamic tides (Wilson \& Power, 2020), future mean-sea-level changes (Sepúlveda et al., 2021), or other sources of prediction errors in nonlinear shallow water models (Bosserelle et al., 2020; Tonini et al., 2021). While sufficient to demonstrate the performance of our offshore-to-onshore Monte-Carlo techniques, the probabilistic inundation results imply large uncertainties in the thrust sources. Reducing these uncertainties would require uncertainty reductions in the offshore PTHA. One way to approach this is via better integration of onshore tsunami observations into the offshore PTHA (e.g. older historical and paleotsunami data). To this end it may be useful to leverage efficient techniques for translating the offshore PTHA onshore, such as proposed herein, which will enable more rigorous comparison of probabilistic models and onshore data.

\section{ACKNOWLEDGEMENTS}

This paper is published with the permission of the CEO of Geoscience Australia. Reviews from Hadi Ghasemi and Jonathan Griffin improved the work. This project was undertaken with the assistance of resources and services from 
the National Computational Infrastructure (NCI), and the Australia Pacific Climate Partnership, which are both supported by the Australian Government. We thank Herve Damlamian for assistance with some elevation datasets, and the Australian Bureau of Meteorology for providing tide-gauge data. The code and tsunami observations used for this study are available at https://github.com/GeoscienceAustralia/ptha/tree/master/misc/monte_ carlo_paper_2021.

\section{References}

Adams, L. M., LeVeque, R. J., \& González, F. I., 2015. The Pattern Method for incorporating tidal uncertainty into probabilistic tsunami hazard assessment (PTHA), Natural Hazards, 76(1), 19-39.

Allgeyer, S. \& Cummins, P., 2014. Numerical tsunami simulation including elastic loading and seawater density stratification, Geophysical Research Letters, 41(7), 2368-2375.

An, C., Liu, H., Ren, Z., \& Yuan, Y., 2018. Prediction of tsunami waves by uniform slip models, Journal of Geophysical Research: Oceans, 123.

Arce Acuña, M. \& Aoki, T., 2018. Tree-based mesh-refinement GPU-accelerated tsunami simulator for real-time operation, Natural Hazards and Earth System Sciences, 18(9), 2561-2602.

Baba, T., Allgeyer, S., Hossen, J., Cummins, P. R., Tsushima, H., Imai, K., Yamashita, K., \& Kato, T., 2017. Accurate numerical simulation of the far-field tsunami caused by the 2011 Tohoku earthquake, including the effects of Boussinesq dispersion, seawater density stratification, elastic loading, and gravitational potential change, Ocean Modelling, 111, 46-54.

Basili, R., Brizuela, B., Herrero, A., Iqbal, S., Lorito, S., Maesano, F. E., Murphy, S., Perfetti, P., Romano, F., Scala, A., Selva, J., Taroni, M., Tiberti, M. M., Thio, H. K., Tonini, R., Volpe, M., Glimsdal, S., Harbitz, C. B., Løvholt, F., Baptista, M. A., Carrilho, F., Matias, L. M., Omira, R., Babeyko, A., Hoechner, A., Gürbüz, M., Pekcan, O., Yalçıner, A., Canals, M., Lastras, G., Agalos, A., Papadopoulos, G., Triantafyllou, I., Benchekroun, S., Agrebi Jaouadi, H., Ben Abdallah, S., Bouallegue, A., Hamdi, H., Oueslati, F., Amato, A., Armigliato, A., Behrens, J., Davies, G., Di Bucci, D., Dolce, M., Geist, E., Gonzalez Vida, J. M., González, M., Macías Sánchez, J., Meletti, C., Ozer Sozdinler, C., Pagani, M., Parsons, T., Polet, J., Power, W., Sørensen, M., \& Zaytsev, A., 2021. The Making of the NEAM Tsunami Hazard Model 2018 (NEAMTHM18), Frontiers in Earth Science, 8, 753.

Behrens, J., Løvholt, F., Jalayer, F., Lorito, S., Salgado-Gálvez, M. A., Sørensen, M., Abadie, S., Aguirre-Ayerbe, I., Aniel-Quiroga, I., Babeyko, A., Baiguera, M., Basili, R., Belliazzi, S., Grezio, A., Johnson, K., Murphy, S., Paris, R., Rafliana, I., De Risi, R., Rossetto, T., Selva, J., Taroni, M., Del Zoppo, M., Armigliato, A., Bureš, V., Cech, P., Cecioni, C., Christodoulides, P., Davies, G., Dias, F., Bayraktar, H. B., González, M., Gritsevich, M., Guillas, S., Harbitz, C. B., Kânoğlu, U., Macías, J., Papadopoulos, G. A., Polet, J., Romano, F., Salamon, A., Scala, A., Stepinac, M., Tappin, D. R., Thio, H. K., Tonini, R., Triantafyllou, I., Ulrich, T., Varini, E., Volpe, M., \& Vyhmeister, E., 2021. Probabilistic tsunami hazard and risk analysis: A review of research gaps, Frontiers in Earth Science, 9, 114.

Berryman, K., Wallace, L., Hayes, G., Bird, P., Wang, K., Basili, R., Lay, T., Pagani, M., Stein, R., Sagiya, T., Rubin, C., Barreintos, S., Kreemer, C., Litchfield, N., Stirling, M., Gledhill, K., Haller, K., \& Costa, C., 2015. The GEM Faulted Earth Subduction Interface Characterisation Project: Version 2.0 - April 2015, Tech. rep., GEM.

Bird, P., 2003. An updated digital model of plate boundaries, Geochemistry Geophysics Geosystems, 4(3), 1-52.

Bletery, Q., Thomas, A. M., Rempel, A. W., Karlstrom, L., Sladen, A., \& De Barros, L., 2016. Mega-earthquakes rupture flat megathrusts, Science, 354(6315), 1027-1031.

Bolker, B. M., 2008. Ecological Models and Data in R, Princeton University Press, Princeton, NJ.

Bosserelle, C., Williams, S., Cheung, K. F., Lay, T., Yamazaki, Y., Simi, T., Roeber, V., Lane, E., Paulik, R., \& Simanu, L., 2020. Effects of Source Faulting and Fringing Reefs on the 2009 South Pacific Tsunami Inundation in Southeast Upolu, Samoa, Journal of Geophysical Research: Oceans, 125(12), e2020JC016537.

Boswood, P. K., 2013. Tsunami Modelling along the East Queensland Coast, in Australasian Coasts and Ports 2013, At Manly, NSW, Australia. 
Brent, R., 1973. Algorithms for Minimization without Derivatives., Englewood Cliffs, NJ: Prentice-Hall.

Burbidge, D., Cummins, P., Mleczko, R., \& Thio, H., 2008. A Probabilistic Tsunami Hazard Assessment for Western Australia, Pure and Applied Geophysics, 165, 2059-2088.

Burbidge, D., Cummins, P., Mleczko, R., Latief, H., Mokhtari, M., Natawidjaja, D., Rajendran, C., \& Thomas, C., 2009. A Probabilistic Tsunami Hazard Assessment of the Indian Ocean Nations, Tech. rep., Geoscience Australia.

Cardno, 2013. NSW Tsunami Inundation Modelling and Risk Assessment, Tech. rep., NSW State Emergency Service and the Office of Environment and Heritage.

Chock, G., 2015. The ASCE 7 Tsunami Loads and Effects Design Standard for the United States, in Handbook of Coastal Disaster Mitigation for Engineers and Planners, pp. 437-460, Elsevier.

Chock, G., Carden, L., Robertson, I., Olsen, M., \& Yu, G., 2013. Tohoku tsunami-induced building failure analysis with implications for u.s. tsunami and seismic design codes, Earthquake Spectra, 29(S1), S99-S126.

Chock, G. Y. K., 2016. Design for tsunami loads and effects in the ASCE 7-16 standard, Journal of Structural Engineering, 142(11), 04016093.

Damlamian, H., Cummins, P., Tokavou, N., Buikoto, L., 'Aho1, L., Sagar, S., Raj, A., \& Powers-Tora, M., 2013. Tsunami Inundation Modelling Of Tongatapu, Kingdom of Tonga, URL: https://www.preventionweb.net/files/45270_219.pdf.

Davies, G., 2019. Tsunami variability from uncalibrated stochastic earthquake models: tests against deep ocean observations 2006-2016, Geophysical Journal International, 218(3), 1939-1960.

Davies, G. \& Griffin, J., 2018. The 2018 Australian Probabilistic Tsunami Hazard Assessment: Hazards from earthquake generated tsunamis., Tech. rep., Geoscience Australia Record 2018/41.

Davies, G. \& Griffin, J., 2020. Sensitivity of Probabilistic Tsunami Hazard Assessment to Far-Field Earthquake Slip Complexity and Rigidity Depth-Dependence: Case Study of Australia, Pure and Applied Geophysics, 177, $1521-1548$.

Davies, G. \& Roberts, S., 2015. Open source flood simulation with a 2D discontinuous-elevation hydrodynamic model, in Proceedings of MODSIM 2015, Gold Coast.

Davies, G., Griffin, J., Løvholt, F., Glimsdal, S., Harbitz, C., Thio, H. K., Lorito, S., Basili, R., Selva, J., Geist, E., \& Baptista, M. A., 2017. A global probabilistic tsunami hazard assessment from earthquake sources, Geological Society, London, Special Publications.

Davies, G., Romano, F., \& Lorito, S., 2020. Global Dissipation Models for Simulating Tsunamis at Far-Field Coasts up to 60 hours Post-Earthquake: Multi-Site Tests in Australia, Frontiers in Earth Science, 8, 497.

de Baar, J. H. S. \& Roberts, S. G., 2017. Multifidelity Sparse-Grid-Based Uncertainty Quantification for the Hokkaido Nansei-oki Tsunami, Pure and Applied Geophysics, 174(8), 3107-3121.

de la Asunción, M., Castro, M. J., Fernández-Nieto, E., Mantas, J. M., Acosta, S. O., \& González-Vida, J. M., 2013. Efficient GPU implementation of a two waves TVD-WAF method for the two-dimensional one layer shallow water system on structured meshes, Computers 83 Fluids, 80, 441 - 452, Selected contributions of the 23rd International Conference on Parallel Fluid Dynamics ParCFD2011.

De Risi, R. \& Goda, K., 2017. Simulation-based probabilistic tsunami hazard analysis: Empirical and robust hazard predictions, Pure and Applied Geophysics, 174(8), 3083-3106.

Deelstra, G., Dhaene, J., \& Vanmaele, M., 2009. An overview of comonotonicity and its applications in finance and insurance, in Advanced Mathematical Methods for Finance, Springer.

Fox-Kemper, B., H. T. H., Xiao, C., Aoalgeirsdottir, G., Drijfhout, S. S., Edwards, T. L., Golledge, N. R., Hemer, M., Kopp, R. E., Krinner, G., Mix, A., Notz, D., Nowicki, S., Nurhati, I. S., Ruiz, L., Sallée, J.-B., Slangen, A. B. A., \& Yu, Y., 2021. Ocean, cryosphere and sea level change., in Climate Change 2021: The Physical Science Basis. Contribution of Working Group I to the Sixth Assessment Report of the Intergovernmental Panel on Climate Change, eds Masson-Delmotte, V., P. Z., Pirani, A., Connors, S. L., Pean, C., Berger, S., Caud, N., Chen, Y., Goldfarb, L., Gomis, M. I., Huang, M., Leitzell, K., Lonnoy, E., Matthews, J. B. R., Maycock, T. K., Waterfield, T., Yelekçi, O., Yu, R., \& Zhou, B., Cambridge University Press. 
Frohlich, C., Hornbach, M. J., Taylor, F. W., Shen, C.-C., Moala, '., Morton, A. E., \& Kruger, J., 2009. Huge erratic boulders in Tonga deposited by a prehistoric tsunami, Geology, 37(2), 131-134.

Fukutani, Y., Suppasri, A., \& Imamura, F., 2018. Quantitative Assessment of Epistemic Uncertainties in Tsunami Hazard Effects on Building Risk Assessments, Geosciences, 8(1).

Fukutani, Y., Moriguchi, S., Terada, K., \& Otake, Y., 2021. Time-dependent probabilistic tsunami inundation assessment using mode decomposition to assess uncertainty for an earthquake scenario, Journal of Geophysical Research: Oceans, 126(7).

Gao, S., Collecutt, G., Syme, W. J., \& Ryan, P., 2020. High resolution numerical modelling of tsunami inundation using quadtree method and GPU acceleration, in Proceedings of the 22nd IAHR-APD Congress 2020, Sapporo, Japan.

Geist, E., 2002. Complex earthquake rupture and local tsunamis., Journal of Geophysical Research, 107(B5).

Geweke, J., 1989. Bayesian Inference in Econometric Models Using Monte Carlo Integration, Econometrica, 57(6), 1317.

Gibbons, S. J., Lorito, S., Macías, J., Løvholt, F., Selva, J., Volpe, M., Sánchez-Linares, C., Babeyko, A., Brizuela, B., Cirella, A., Castro, M. J., de la Asunción, M., Lanucara, P., Glimsdal, S., Lorenzino, M. C., Nazaria, M., Pizzimenti, L., Romano, F., Scala, A., Tonini, R., Manuel González Vida, J., \& Vöge, M., 2020. Probabilistic tsunami hazard analysis: High performance computing for massive scale inundation simulations, Frontiers in Earth Science, 8, 623 .

Giles, D., Gopinathan, D., Guillas, S., \& Dias, F., 2021. Faster than real time tsunami warning with associated hazard uncertainties, Frontiers in Earth Science, 8, 560.

Goff, J., 2011. Evidence of a previously unrecorded local tsunami, 13 April 2010, Cook Islands: implications for Pacific Island countries, Natural Hazards and Earth System Sciences, 11(5), 1371-1379.

Goff, J., Lamarche, G., Pelletier, B., Chagué-Goff, C., \& Strotz, L., 2011. Predecessors to the 2009 South Pacific tsunami in the Wallis and Futuna archipelago, Earth-Science Reviews, 107(1-2), 91-106.

Goff, J., Witter, R., Terry, J., \& Spiske, M., 2020. Palaeotsunamis in the Sino-Pacific region, Earth-Science Reviews, 210, 103352.

González, M., Álvarez-Gómez, J. A., Aniel-Quiroga, Í., Otero, L., Olabarrieta, M., Omira, R., Luceño, A., Jelinek, R., Krausmann, E., Birkman, J., Baptista, M. A., Miranda, M., \& Aguirre-Ayerbe, I., 2021. Probabilistic Tsunami Hazard Assessment in Meso and Macro Tidal Areas. Application to the Cádiz Bay, Spain, Frontiers in Earth Science, $\mathbf{9}$.

Goto, C. \& Ogawa, Y., 1997. Numerical method of tsunami simulation with the leap-frog scheme, Tech. rep., IUGG/IOC Time Project.

Grezio, A., Babeyko, A., Baptista, M. A., Behrens, J., Costa, A., Davies, G., Geist, E. L., Glimsdal, S., González, F. I., Griffin, J., Harbitz, C. B., LeVeque, R. J., Lorito, S., Løvholt, F., Omira, R., Mueller, C., Paris, R., Parsons, T., Polet, J., Power, W., Selva, J., Sørensen, M. B., \& Thio, H. K., 2017. Probabilistic tsunami hazard analysis: Multiple sources and global applications, Reviews of Geophysics, 55(4), 1158-1198, 2017RG000579.

Guillas, S., Sarri, A., Day, S. J., Liu, X., \& Dias, F., 2018. Functional emulation of high resolution tsunami modelling over Cascadia, Ann. Appl. Stat., 12(4), 2023-2053.

Hayes, G. P., Moore, G. L., Portner, D. E., Hearne, M., Flamme, H., Furtney, M., \& Smoczyk, G. M., 2018. Slab2, a comprehensive subduction zone geometry model, Science.

Heuret, A., Conrad, C. P., Funiciello, F., Lallemand, S., \& Sandri, L., 2012. Relation between subduction megathrust earthquakes, trench sediment thickness and upper plate strain, , 39, L05304.

Hoechner, A., Babeyko, A. Y., \& Zamora, N., 2016. Probabilistic tsunami hazard assessment for the Makran region with focus on maximum magnitude assumption, Natural Hazards and Earth System Sciences, 16, 1339-1350.

Kalligeris, N., Montoya, L., Ayca, A., \& Lynett, P., 2017. An approach for estimating the largest probable tsunami from far-field subduction zone earthquakes, Natural Hazards, 89, 233. 
Lamarche, G., Popinet, S., Pelletier, B., Mountjoy, J., Goff, J., Delaux, S., \& Bind, J., 2015. Scenario-based numerical modelling and the palaeo-historic record of tsunamis in Wallis and Futuna, Southwest Pacific, Natural Hazards and Earth System Sciences, 15(8), 1763-1784.

Lane, E. M., Gillibrand, P. A., Wang, X., \& Power, W., 2012. A Probabilistic Tsunami Hazard Study of the Auckland Region, Part II: Inundation Modelling and Hazard Assessment, Pure and Applied Geophysics, 170(9-10), 16351646.

Lay, T., Ammon, C. J., Kanamori, H., Rivera, L., Koper, K. D., \& Hutko, A. R., 2010. The 2009 Samoa-Tonga great earthquake triggered doublet, Nature, 466, 964-968.

Leonard, G., Power, W., Lukovic, B., Smith, W., Johnston, D., \& Downes, G., 2008. Tsunami evacuation zones for Wellington and Horizons regions defined by a GIS-calculated attenuation rule, Tech. rep., GNS Science Report 2008/30, 22pp.

Li, H., Yuan, Y., Xu, Z., Wang, Z., Wang, J., Wang, P., Gao, Y., Hou, J., \& Shan, D., 2016a. The Dependency of Probabilistic Tsunami Hazard Assessment on Magnitude Limits of Seismic Sources in the South China Sea and Adjoining Basins, Pure and Applied Geophysics, 174(6), 2351-2370.

Li, L., Switzer, A. D., Chan, C.-H., Wang, Y., Weiss, R., \& Qiu, Q., 2016b. How heterogeneous coseismic slip affects regional probabilistic tsunami hazard assessment: A case study in the South China Sea, Journal of Geophysical Research: Solid Earth, 121(8), 6250-6272, 2016JB013111.

Li, L., Switzer, A. D., Wang, Y., Chan, C.-H., Qiu, Q., \& Weiss, R., 2018. A modest 0.5-m rise in sea level will double the tsunami hazard in Macau, Science Advances, 4(8), eaat1180.

Lorito, S., Selva, J., Basili, R., Romano, F., Tiberti, M., \& Piatanesi, A., 2015. Probabilistic hazard for seismically induced tsunamis: accuracy and feasibility of inundation maps, Geophysical Journal International, 200, 574-588.

Løvholt, F., Fraser, S., Salgado-Gálvez, M., Lorito, S., J., S., F., R., Suppasri, A., Mas, E., Polet, J., Behrens, J., Canals, M., Papadopoulos, G. A., Schaefer, A., Zamora, N., Chacon, S., Wood, N., Aguirre-Ayerbe, I., AnielQuiroga Zorilla, I., Gonzalez Rodriguez, M., Johnson, D., Leonard, G., Paris, R., Guillas, S., Dias, F., \& Baptista, M., 2019. Global trends in advancing tsunami science for improved hazard and risk understanding. contributing paper to gar 2019, in Global Assessment Report on Disaster Risk Reduction..

Lynett, P., Wei, Y., \& Arcas, D., 2016. Tsunami hazard assessment: Best modeling practices and state-of-the-art technology, Tech. rep., Office of Nuclear Regulatory Research.

Lynett, P. J., Gately, K., Wilson, R., Montoya, L., Arcas, D., Aytore, B., Bai, Y., Bricker, J. D., Castro, M. J., Cheung, K. F., David, C. G., Dogan, G. G., Escalante, C., González-Vida, J. M., Grilli, S. T., Heitmann, T. W., Horrillo, J., Kânoğlu, U., Kian, R., Kirby, J. T., Li, W., Macías, J., Nicolsky, D. J., Ortega, S., Pampell-Manis, A., Park, Y. S., Roeber, V., Sharghivand, N., Shelby, M., Shi, F., Tehranirad, B., Tolkova, E., Thio, H. K., Velioğlu, D., Yalçıner, A. C., Yamazaki, Y., Zaytsev, A., \& Zhang, Y., 2017. Inter-model analysis of tsunami-induced coastal currents, Ocean Modelling, 114, 14-32.

Macías, J., Castro, M. J., Ortega, S., \& González-Vida, J. M., 2020. Performance assessment of Tsunami-HySEA model for NTHMP tsunami currents benchmarking. Field cases, Ocean Modelling, 152, 101645.

Makinoshima, F., Oishi, Y., Yamazaki, T., Furumura, T., \& Imamura, F., 2021. Early forecasting of tsunami inundation from tsunami and geodetic observation data with convolutional neural networks, Nature Communications, 12(1).

McCaffrey, R., 2008. Global frequency of magnitude 9 earthquakes, Geology, 36(3), 263-266.

MCDEM, 2016. Tsunami evacuation zones: Director's guideline for civil defence emergency management groups, Tech. rep., Ministry of Civil Defence \& Emergency Management.

Melgar, D., Williamson, A. L., \& Salazar-Monroy, E. F., 2019. Differences between heterogenous and homogenous slip in regional tsunami hazards modelling, Geophysical Journal International, 219(1), 553-562.

Miranda, J. M., Baptista, M. A., \& Omira, R., 2014. On the use of Green's summation for tsunami waveform estimation: a case study, Geophysical Journal International, 199(1), 459-464. 
Molinari, I., Tonini, R., Lorito, S., Piatanesi, A., Romano, F., Melini, D., Hoechner, A., Gonzàlez Vida, J. M., Maciás, J., Castro, M. J., \& de la Asunción, M., 2016. Fast evaluation of tsunami scenarios: uncertainty assessment for a Mediterranean Sea database, Natural Hazards and Earth System Sciences, 16(12), 2593-2602.

Mueller, C., Power, W., Fraser, S., \& Wang, X., 2015. Effects of rupture complexity on local tsunami inundation: Implications for probabilistic tsunami hazard assessment by example, Journal of Geophysical Research (Solid Earth), 120, 488-502.

NTHMP, 2012. Proceedings and Results of the 2011 NTHMP Model Benchmarking Workshop, Tech. rep., National Tsunami Hazard Mitigation Program, NOAA Special Report.

Okal, E. A., 2011. Tsunamigenic Earthquakes: Past and Present Milestones, Pure and Applied Geophysics, 168, 969-995.

Okal, E. A., 2015. The quest for wisdom: lessons from 17 tsunamis, 2004-2014, Philosophical Transactions of the Royal Society A: Mathematical, Physical and Engineering Sciences, 373(2053), 20140370.

Okal, E. A., Borrero, J., \& Synolakis, C. E., 2004. The earthquake and tsunami of 1865 November 17: evidence for far-field tsunami hazard from Tonga, Geophysical Journal International, 157, 164-174.

Okal, E. A., Borrero, J. C., \& Chagué-Goff, C., 2011. Tsunamigenic predecessors to the 2009 Samoa earthquake, Earth Science Reviews, 107, 128-140.

Owen, A. \& Zhou, Y., 2000. Safe and effective importance sampling, Journal of the American Statistical Association, 95(449), 135-143.

Park, H., Cox, D. T., Lynett, P. J., Wiebe, D. M., \& Shin, S., 2013. Tsunami inundation modeling in constructed environments: A physical and numerical comparison of free-surface elevation, velocity, and momentum flux, Coastal Engineering, 79, 9-21.

Plescia, S. M. \& Hayes, G. P., 2020. Geometric controls on megathrust earthquakes, Geophysical Journal International, 222(2), 1270-1282.

Power, W., Wallace, L., Wang, X., \& Reyners, M., 2012. Tsunami Hazard Posed to New Zealand by the Kermadec and Southern New Hebrides Subduction Margins: An Assessment Based on Plate Boundary Kinematics, Interseismic Coupling, and Historical Seismicity, Pure and Applied Geophysics, 169(1), 1-36.

Power, W., Clark, K., King, D. N., Borrero, J., Howarth, J., Lane, E. M., Goring, D., Goff, J., Chagué-Goff, C., Williams, J., Reid, C., Whittaker, C., Mueller, C., Williams, S., Hughes, M. W., Hoyle, J., Bind, J., Strong, D., Litchfield, N., \& Benson, A., 2017. Tsunami runup and tide-gauge observations from the 14 November 2016 M7.8 Kaikōura earthquake, New Zealand, Pure and Applied Geophysics.

Power, W., Wang, X., Wallace, L., Clark, K., \& Mueller, C., 2017. The New Zealand Probabilistic Tsunami Hazard Model: development and implementation of a methodology for estimating tsunami hazard nationwide, Geological Society, London, Special Publications, 456.

Romano, F., Gusman, A. R., Power, W., Piatanesi, A., Volpe, M., Scala, A., \& Lorito, S., 2021. Tsunami Source of the 2021 MW 8.1 Raoul Island Earthquake from DART and Tide-gauge data inversion, Geophysical Research Letters, n/a(n/a), e2021GL094449, e2021GL094449 2021GL094449.

Rong, Y., Jackson, D. D., Magistrale, H., \& Goldfinger, C., 2014. Magnitude limits of subduction zone earthquakes, Bulletin of the Seismological Society of America, 104(5), 2359-2377.

Scholz, C. H. \& Campos, J., 2012. The seismic coupling of subduction zones revisited, Journal of Geophysical Research, 117(B05310).

Sepúlveda, I., Liu, P. L.-F., Grigoriu, M., \& Pritchard, M., 2017. Tsunami Hazard Assessments with Consideration of Uncertain Earthquake Slip Distribution and Location, Journal of Geophysical Research: Solid Earth.

Sepúlveda, I., Liu, P. L.-F., \& Grigoriu, M., 2019. Probabilistic tsunami hazard assessment in south china sea with consideration of uncertain earthquake characteristics, Journal of Geophysical Research: Solid Earth, 124(1), 658-688. 
Sepúlveda, I., Haase, J. S., Liu, P. L.-F., Grigoriu, M., \& Winckler, P., 2021. Non-stationary probabilistic tsunami hazard assessments incorporating climate-change-driven sea level rise, Earth's Future, 9(6), e2021EF002007.

Tang, L., Titov, V. V., Wei, Y., Mofjeld, H. O., Spillane, M., Arcas, D., Bernard, E. N., Chamberlin, C., Gica, E., \& Newman, J., 2008. Tsunami forecast analysis for the May 2006 Tonga tsunami, Journal of Geophysical Research: Oceans, 113(C12).

Tang, L., Titov, V. V., \& Chamberlin, C. D., 2009. Development, testing, and applications of site-specific tsunami inundation models for real-time forecasting, Journal of Geophysical Research, 114(C12025).

Thompson, S. K., 2012. Sampling, Third Edition., John Wiley \& Sons, Inc.

Tokdar, S. T. \& Kass, R. E., 2009. Importance sampling: a review, WIREs Computational Statistics, 2(1), 54-60.

Tonini, R., Di Manna, P., Lorito, S., Selva, J., Volpe, M., Romano, F., Basili, R., Brizuela, B., Castro, M. J., de la Asunción, M., Di Bucci, D., Dolce, M., Garcia, A., Gibbons, S. J., Glimsdal, S., González-Vida, J. M., Løvholt, F., Macías, J., Piatanesi, A., Pizzimenti, L., Sánchez-Linares, C., \& Vittori, E., 2021. Testing Tsunami Inundation Maps for Evacuation Planning in Italy, Frontiers in Earth Science, 9, 82.

UNESCO/IOC, 2020. Experts Meeting on Tsunami Sources, Hazards, Risk and Uncertainties Associated with the Tonga-Kermadec Subduction Zone, Tech. rep., UNESCO/IOC.

Volpe, M., Lorito, S., Selva, J., Tonini, R., Romano, F., \& Brizuela, B., 2019. From regional to local SPTHA: efficient computation of probabilistic tsunami inundation maps addressing near-field sources, Natural Hazards and Earth System Sciences, 19(3), 455-469.

Watada, S., Kusumoto, S., \& Satake, K., 2014. Traveltime delay and initial phase reversal of distant tsunamis coupled with the self-gravitating elastic earth, Journal of Geophysical Research: Solid Earth, 119(5), 4287-4310.

Whiteway, T., 2009. Australian Bathymetry and Topography Grid, June 2009, Tech. rep., Geoscience Australia Record 2009/21.

Williamson, A. L., Rim, D., Adams, L. M., LeVeque, R. J., Melgar, D., \& González, F. I., 2020. A source clustering approach for efficient inundation modeling and regional scale probabilistic tsunami hazard assessment, Frontiers in Earth Science, 8.

Wilson, K. M. \& Power, H. E., 2020. Tsunami modelling with static and dynamic tides in drowned river valleys with morphological constrictions, Pure and Applied Geophysics.

Wood, N., Peters, J., Wilson, R., Sherba, J., \& Henry, K., 2020. Variations in community evacuation potential related to average return periods in probabilistic tsunami hazard analysis, International Journal of Disaster Risk Reduction, 50, 101871.

Zamora, N., Catalán, P. A., Gubler, A., \& Carvajal, M., 2021. Microzoning tsunami hazard by combining flow depths and arrival times, Frontiers in Earth Science, 8, 747.

Zöller, G., 2013. Convergence of the frequency-magnitude distribution of global earthquakes: Maybe in 200 years, Geophysical Research Letters, 40(15), 3873-3877.

Zöller, G., 2017. Comment on "Estimation of Earthquake Hazard Parameters from Incomplete Data Files. Part III. Incorporation of Uncertainty of Earthquake-Occurrence Model" by Andrzej Kijko, Ansie Smit, and Markvard A. Sellevoll, Bulletin of the Seismological Society of America, 107(4), 1975. 